\title{
Externalities, Potential, Value and Consistency
}

\author{
Bhaskar Dutta, Lars Ehlers, Anirban Kar*
}

August 18, 2010

\begin{abstract}
We provide new characterization results for the value of games in partition function form. In particular, we use the potential of a game to define the value. We also provide a characterization of the class of values which satisfies one form of reduced game consistency. form.

Keywords: Shapley value, potential, consistency, games in partition function
\end{abstract}

${ }^{*}$ Dutta is in the Department of Economics, University of Warwick, Coventry CV4 7AL, UK. Ehlers is in the Département de Sciences Économiques, Université de Montréal, Québec, Canada. Kar is in the Department of Economics, Delhi School of Economics, University of Delhi, India. Ehlers acknowledges financial support from the SSHRC (Canada), the FQRSC (Québec), and the hospitality of Harvard Business School where this paper was finished. Kar acknowledges Department of Economics, University of Warwick, where he was a faculty member when this paper was initially drafted. We are especially grateful to the referee and the associate editor for their detailed and helpful comments and suggestions. 


\section{Introduction}

In a variety of economic and social contexts, the activities of one group of agents affect payoffs of other groups. Consider, for instance, the issue of political alliances between different groups of countries. The benefit to each group will typically depend on the strength of the alliance between opposing groups of countries. Similarly, the benefits to one group of agents from activities aimed at controlling pollution depend upon whether other agents are also engaged in similar pollution abatement exercises.

In a framework where such externalities across coalitions or groups are absent, Shapley [15] provided what has become the focal method for distributing the surplus generated by cooperation amongst groups of agents. Shapley obtained a remarkable uniqueness result by showing that there is only one solution - the Shapley value which satisfies some seemingly mild axioms. ${ }^{1}$ The Shapley value essentially gives each player the average of his marginal contributions to different coalitions

There have been other axiomatic characterizations of the Shapley value. For instance, Young [19] uses a monotonicity principle which states that if a game changes so that some player's contribution to all coalitions increases or stays the same then the player's reward should not decrease. Young shows that the Shapley value is the only efficient and symmetric solution that is monotonic in this sense.

Hart and Mas-Colell [7] provide two derivations of the Shapley value. First, they use the analytical tool of a potential function to formalise the notion of rewarding players according to marginal productivity. The potential function has the property that the sum of the players' marginal products (according to the potential) adds up to the worth of the grand coalition. Moreover, the Shapley value happens to coincide with the vector of marginal products. Thus, this provides another very interesting interpretation of the value. Hart and Mas-Colell also define an internal consistency property of solution concepts and show that the Shapley value is the unique solution satisfying this consistency property and the so-called standard property on two-person games. $^{2}$

Given the widespread presence of externalities, it is important to study the distributional issue in environments with externalities. A game in partition function form,${ }^{3}$ in which the "worth" of any coalition depends on how players outside the coalition are organised, provides an appropriate framework within which one can describe solution concepts for games with externalities. Not surprisingly, this has received some recent attention. For instance, Macho-Stadler et al. [6] provide characterizations of extensions of the Shapley value to partition function games, using axioms which are designed to capture the intuitive content of Shapley's original axioms. ${ }^{4}$ In contrast, de Clippel and Serrano [5] follow the approach of Young [19],

\footnotetext{
${ }^{1}$ Sergiu Hart provides on his website a large and useful bibliography of value theory in cooperative games.

${ }^{2}$ This property states that on two-person games, the gains from cooperation be split equally between the two players.

${ }^{3}$ This is due to Thrall and Lucas [18]. See also Ray [14] for a discussion of games in partition function form.

${ }^{4}$ Bolger [3] and Myerson [10] are earlier contributions along the same lines.
} 
and also provide alternative characterizations of value concepts for partition function games. Other recent contributions include Albizuri et al. [1], Fujinaka [5] and Pham Do and Norde [13].

In this paper, we follow the methodology of Hart and Mas-Colell [7]. The potential approach requires that "subgames" be well-defined for each player set. This is a trivial issue for characteristic function games since a subgame is simply the "projection" of the original game to the appropriate player set. For games in partition function form there is no such unambiguous answer since the worth of each coalition depends on how the complementary coalition is partitioned. However, it is natural to require that in a subgame the worth of a coalition for a given partition is some function of the worths of the coalition and the partitions where the absent players join the given partition in some way. Such functions could take averages or incorporate optimistic/pessimistic expectations.

We adopt the following procedure. We define restriction operators, which are functions that "project" each partition function game on any set of players $N$ to its subgames with player sets $N \backslash\{i\}$. More precisely, each restriction operator determines the worth of a coalition for a given partition in the subgame via aggregating the worths of this coalition and the partitions resulting from $i$ joining one of the existing coalitions in the original game. Subgames so constructed are "estimates" or "approximations" based on the available data. A basic requirement on restriction operators is that the order in which players are removed from a game should be irrelevant for the restricted game. In other words, first removing player 1 and then player 2 or first removing player 2 and then player 1 should result in the same game restricted to the player set without 1 and 2. We call this requirement path-independence. Our first main result shows that any path-independent restriction operator defines uniquely a potential for games in partition function form. This unique potential coincides with that of a particular characteristic function game without externalities. A natural step is to define the value of the partition function game to be the Shapley value of this game in characteristic function form. For any path-independent restriction operator $r$, we call this value the $r$-Shapley value.

We give examples to illustrate that a rich class of restriction operators satisfies path independence. Hence, our first result demonstrates that the potential approach yields a large class of extensions of the Shapley value from games without externalities to games with externalities.

Since Path independence by itself does not have much bite in singling out restriction operators, we go on to impose additional axioms on these operators which are parallel to the ones used by Shapley [15] on solutions. However, we emphasize that our axioms are imposed on the restriction operators and not on the solution concept. This allows us to characterise a one-parameter class of restriction operators. Of course, each operator in our class gives rise to a different value through the potential. We show that all our values satisfy the basic properties of the Shapley value, suitably extended to the more general framework of games with externalities.

We also follow Hart and Mas-Colell [7] by using their "reduced game" consistency condition, suitably adapted to games in partition function form, to characterise 
solution concepts. We show that a large number of solutions satisfy this definition of consistency and the so-called standard property on two-person games.

The plan of this paper is the following. Section 2 describes the general framework and some notation. In Section 3, we introduce restriction operators and the potential approach. This section also contains our characterisation result using path independence. In Section 4, we describe the additional axioms as well as the characterisation result on restriction operators. In Section 5, we show that the solution concepts implied by the class of restriction operators characterised in Section 4 satisfy the natural extensions of the original Shapley axioms to partition function games. Section 6 contains a characterisation result based on consistency. In Section 7, we briefly discuss the existing literature, and also compare the values derived using the potential approach to those derived through other approaches. The Appendix contains the proofs of all our results.

\section{Framework and Notation}

Let $\mathbb{N}$ be some finite set of players, containing at least three players. We are interested in games with externalities where the player set can be any (non-empty) subset of $\mathbb{N}$. We define such games formally.

Choose any subset $N$ of $\mathbb{N}$. A partition of $N$ is a set $\pi=\left\{T_{1}, \ldots, T_{k}\right\}$ such that (i) for all $i, j \in\{1, \ldots, k\}, T_{i} \cap T_{j}=\emptyset$, and (ii) $\cup_{i=1}^{k} T_{i}=N$. Let $\Pi_{N}$ denote the set of all partitions of $N$.

A coalition $S$ is a non-empty subset of $N$. Let $\Pi_{S}$ denote the set of all partitions of coalition $S$. We will use the notation $\pi(S)$ to denote a partition of $S$. For any coalition $S$ and any partition $\pi(N)$, let $S \cap \pi(N)=\{S \cap T: T \in \pi(N)\}$. For any coalition $S, S^{c}$ denotes the set $N \backslash S .{ }^{5}$ For any $S, \pi^{t}(S)=\{\{i\} \mid i \in S\}$. That is, $\pi^{t}(S)$ is the "trivial" partition of $S$ consisting of the singleton members of $S$.

Given player set $N \subseteq \mathbb{N}$, let $(N, v)$ denote a game in partition function form. That is, $v$ specifies a real number for every coalition $S$ and for every partition of $S^{c}$. We represent this as $v\left(S ; \pi\left(S^{c}\right)\right)$, and call this the worth of coalition $S$ when $S^{c}$ is partitioned according to $\pi\left(S^{c}\right)$. We will call $\left(S ; \pi\left(S^{c}\right)\right)$ an embedded coalition. For any player set $N$, we will simply write $v(N)$ instead of $v(N ; \emptyset)$. Abusing notation, we sometimes write $v\left(S ; T_{1}, \ldots, T_{k}\right)$ instead of $v\left(S ;\left\{T_{1}, \ldots, T_{k}\right\}\right)$ when there is no ambiguity.

The game $(N, v)$ is with externalities if the worth of at least one coalition depends on the partition of the other players, i.e. $v\left(S ; \pi\left(S^{c}\right)\right) \neq v\left(S ; \pi^{\prime}\left(S^{c}\right)\right)$ for at least one coalition $S$ and some $\pi\left(S^{c}\right), \pi^{\prime}\left(S^{c}\right) \in \Pi_{S^{c}}$.

A game $(N, v)$ is without externalities if the worth of any coalition $S$ is independent of how the complementary coalition $S^{c}$ is partitioned. That is, a game without externalities is the "traditional" TU game in characteristic form with $v: 2^{N} \rightarrow \mathbb{R}$

\footnotetext{
${ }^{5}$ Since we want to allow for variable player sets, there may be some ambiguity about the notation $S^{c}$. This is to be understood as the complement of the coalition $S$ in the player set under consideration.
} 
(where $2^{N}$ denotes the set of all non-empty subsets of $N$ ). We will typically use $w, w^{\prime}$ etc., to denote games without externalities and $v, v^{\prime}$ to denote games with externalities.

Let $\mathbb{V}$ and $\mathbb{W}$ denote respectively the class of all partition function games and characteristic function games which can be constructed on player sets that are subsets of $\mathbb{N}$. Of course, $\mathbb{W} \subset \mathbb{V}$.

A solution (concept) or value is a mapping $\varphi$ which associates with every game $(N, v)$ in $\mathbb{V}$ a vector in $\mathbb{R}^{|N|}$ satisfying $\sum_{i \in N} \varphi_{i}(N, v)=v(N)$. A value determines the payoffs of the individual players in any game.

\section{The Potential Approach}

The traditional approach in economics of paying individuals according to their marginal productivity has no straightforward analogue in cooperative game theory because the sum of the players' marginal contributions to the grand coalition is typically not a feasible payoff vector. Hart and Mas-Colell [7] develop the potential function as a new analytical tool which helps in formalising the notion of rewarding players according to their marginal contributions. In particular, they define the potential as a real-valued function $P$ on the set of all TU games (without externalities) such that the marginal contributions of all players according to $P$ add up to the worth of the grand coalition. The resulting payoff vector coincides with the Shapley value of the $\mathrm{TU}$ game.

More formally, they define a function $P: \mathbb{W} \rightarrow \mathbb{R}$ which assigns a real number $P(N, w)$ to every TU game $(N, w)$, and define the marginal contribution of player $i$ to be

$$
D^{i} P(N, w)=P(N, w)-P(N \backslash\{i\}, w)
$$

Note that $(N \backslash\{i\}, w)$ is the projection of $(N, w)$ on $N \backslash\{i\}$. The function $P$ is a potential if $P(\emptyset, 0)=0$ and

$$
\sum_{i \in N} D^{i} P(N, w)=w(N)
$$

for every TU game $(N, w)$. Hart and Mas-Colell [7] show that there is a unique potential function and that for every game $(N, w)$, the payoff vector $\left(D^{i} P(N, w)\right)_{i \in N}$ coincides with the Shapley value of the game, i.e.,

$$
D^{i} P(N, w)=S h_{i}(N, w)=\sum_{S \subseteq N . s . t .} \frac{(s-1) !(n-s) !}{n !}[w(S)-w(S \backslash\{i\})]
$$

where $s$ and $n$ denote the cardinalities of the sets $S$ and $N$ respectively.

Our principal goal in this paper is to use the potential approach to derive a value for games in partition function form. Notice that this approach requires us to specify subgames $(N \backslash\{i\}, v)$ for each game $(N, v)$. This is perfectly straightforward for characteristic function games since $(N \backslash\{i\}, w)$ is simply the restriction of $(N, w)$ 
to $N \backslash\{i\}$. Unfortunately, there is no unambiguous way of deriving subgames for games in partition function form.

Consider, for instance, $N=\{1,2,3\}$, and suppose $v(N)=a, v(\{i, j\} ;\{k\})=b$, $v(\{i\} ;\{j, k\})=c$, and $v\left(\{i\} ; \pi^{t}(\{j, k\})\right)=d$. Below we specify all the worths in the game $(N, v)$. We have $v(N)=a$ and

$$
\begin{array}{ll}
v(\{1,2\} ;\{3\})=b, \quad v(\{1\} ;\{2,3\})=c, & v\left(\{1\} ; \pi^{t}(\{2,3\})\right)=d, \\
v(\{1,3\} ;\{2\})=b, & v(\{2\} ;\{1,3\})=c, \quad v\left(\{2\} ; \pi^{t}(\{1,3\})\right)=d, \\
v(\{2,3\} ;\{1\})=b, & v(\{3\} ;\{1,2\})=c, \quad v\left(\{3\} ; \pi^{t}(\{1,2\})\right)=d .
\end{array}
$$

Then, what is $\left(\{2,3\}, v^{-1}\right)$ where $v^{-1}$ denotes the corresponding partition function for the player set $\{2,3\}$. Since there is only one possible partition of $\{1,2,3\}$ in which $\{2,3\}$ is a member, it is natural to define $v^{-1}(\{2,3\}) \equiv v(\{2,3\} ;\{1\}) .{ }^{6}$ The problem appears when one tries to specify $v^{-1}(\{2\} ;\{3\})$ from knowledge of $v$ on the player set $\{1,2,3\}$. Should we take a simple or weighted average of $v(\{2\} ;\{1,3\})$ and $v\left(\{2\} ; \pi^{t}(\{1,3\})\right)$ ? Or take the maximum (or minimum) worth amongst these?

\subsection{Restriction Operators}

We adopt the following procedure. Define a restriction operator to be a mapping $r$ from $\mathbb{V}$ to $\mathbb{V}$ which specifies for each game $(N, v) \in \mathbb{V}$ a "subgame" game $\left(N \backslash\{i\}, v^{-i, r}\right)$ for each $i \in N$. Notice that once a particular restriction operator $r$ has been specified, then it is straightforward to define an $r$-potential function. We will subsequently use $r$-potential functions to derive corresponding values following the Hart and Mas-Colell procedure.

In order to define a restriction operator, we need some notation.

Let $\pi(S)=\left\{S_{1}, S_{2} \ldots, S_{K}\right\}$ be a partition of some set $S$. Then, for any $i \notin S$, $\pi^{+i}(S)$ is the set of partitions of $S \cup\{i\}$ where $i$ either joins one of the coalitions $S_{k}$ of $\pi(S)$, the other coalitions remaining unchanged, or it is the partition $\left\{S_{1}, \ldots, S_{K},\{i\}\right\}$. For instance, if $S=\{1,2,3\}$ and $\pi(S)=\{\{1,2\},\{3\}\}$, then $\pi^{+4}(S)=\{\{\{1,2,4\},\{3\}\} ;\{\{1,2\},\{3,4\}\} ;\{\{1,2\},\{3\},\{4\}\}\}$.

Given any $(N, v)$ in $\mathbb{V}$, the general form of a restriction operator is specified below:

$$
v^{-i, r}\left(S ; \pi\left((S \cup\{i\})^{c}\right)\right)=r_{i, S, \pi\left((S \cup\{i\})^{c}\right)}^{N}\left((v(S ; \pi))_{\pi \in \pi^{+i}\left((S \cup\{i\})^{c}\right)}\right)
$$

For instance, if $\left.\pi\left((S \cup\{i\})^{c}\right)\right)=\left\{S_{1}, \ldots, S_{K}\right\}$, then

$$
\begin{gathered}
v^{-i, r}\left(S ; \pi\left((S \cup\{i\})^{c}\right)\right) \quad= \\
r_{i, S, \pi\left((S \cup\{i\})^{c}\right)}^{N}\left(v\left(S ; S_{1} \cup\{i\}, S_{2}, \ldots, S_{K}\right), \ldots, v\left(S ; S_{1}, \ldots, S_{K} \cup\{i\}\right), v\left(S ; S_{1}, S_{2}, \ldots, S_{K},\{i\}\right)\right) .
\end{gathered}
$$

So, the worth of any coalition $S$ corresponding to $\left(S ; \pi\left((S \cup\{i\})^{c}\right)\right)$ in the subgame when player $i$ is absent is some function of the worths $(S ; \pi)$ where $\pi$ is some element of $\pi^{+i}\left((S \cup\{i\})^{c}\right)$. For instance, if $N=\{1,2,3,4\}$, then $\left.v^{-4, r}(\{1\} ;\{2,3\}\}\right)=$

\footnotetext{
${ }^{6}$ In general, one can specify $v^{-i}(N \backslash\{i\})=v(N \backslash\{i\} ;\{i\})$.
} 
$r_{4,\{1\},\{2,3\}}^{N}(v(\{1\} ;\{2,3,4\}), v(\{1\} ;\{2,3\},\{4\}))$. In the subgame the worth of any coalition $S$ for a specific partition of the other players depends only on the worths of $S$ where player $i$ joins one of the existing members of the given partition or remains alone. Note that the order in which the variables appear in the restriction operator is fixed.

Of course, this specification hardly imposes any restriction on the $r_{i, S, \pi\left((S \cup\{i\})^{c}\right)}^{N}$ functions, and hence on the restriction operators. For instance, the specification allows $r_{i, S, \pi\left((S \cup\{i\})^{c}\right)}^{N}$ to depend on $i$, the coalition $S$, the partition of its complement in the subgame, and the original player set. In order to make notation simpler, we will typically drop the superscript $N$ and the subscripts $i, S$ and $\pi\left((S \cup\{i\})^{c}\right)$ whenever no confusion can result from this and write $r$ instead of $r_{i, S, \pi\left((S \cup\{i\})^{c}\right)^{N}}^{N}$ Similarly, we will drop the superscript $r$ and write $v^{-i}$ instead of $v^{-i, r}$.

We use an axiomatic approach to restriction operators. Each axiom is meant to be a "reasonable" property of a restriction operator. As we proceed, we will impose more and more axioms on restriction operators and finally single out a one-parameter class of restriction operators via axioms which are parallel to two axioms used by Shapley in his characterization of the Shapley value.

The most basic axiom is that of Path Independence. For any $i, j \in N$, let $v^{-i j}=$ $\left(v^{-i}\right)^{-j}$.

Definition 1 A restriction operator $r$ satisfies Path Independence (PI) if for all $(N, v) \in \mathbb{V}$, for all $i, j \in N, v^{-i j}=v^{-j i}$.

If Path Independence is not satisfied, then the subgame on the player set $N \backslash\{i, j\}$ is not well-defined. So, Path Independence is almost a necessary condition to use the potential approach since the latter requires well-defined subgames. In particular, then the subgame $v^{-S}$, where some coalition $S \subset N$ leaves the game $v$, is well-defined: under Path Independence the players belonging to $S$ are sequentially removed from $v$ in any arbitrary order. Note that Path Independence implies that the subgame $v^{-S}$ derived by removing individual players in $S$ one a time would coincide with the subgame on $S^{c}$ obtained by removing disjoint subsets of $S$ - say $S_{1}$ and $S_{2}$ in some order.

Given any well-defined restriction operator $r$, it is now easy to define the $r$ potential function of any game $(N, v)$ in $\mathbb{V}$. More formally, we define a function $P^{r}: \mathbb{V} \rightarrow \mathbb{R}$ which assigns a real number $P^{r}(N, v)$ to every game $(N, v)$. The marginal contribution of player $i$ is

$$
D^{i} P^{r}(N, v)=P^{r}(N, v)-P^{r}\left(N \backslash\{i\}, v^{-i}\right)
$$

The function $P^{r}$ is an $r$-potential if $P^{r}(\emptyset, 0)=0$ and

$$
\sum_{i \in N} D^{i} P^{r}(N, v)=v(N)
$$

for all games $(N, v)$ in $\mathbb{V}$. Of course, once a restriction operator is fixed, the potential function is uniquely defined. 
Path independence by itself has an important implication for the subsequent analysis. In particular, it allows us to relate the $r$-potential of any game with externalities to the potential of a game without externalities. Put differently, we can derive a characteristic function game from any game in partition function form, and then define the value of the partition function form game to be the Shapley value of the associated characteristic function game. Of course, this has also been the approach followed by other recent contributions. ${ }^{7}$ The novelty of our approach is that we use the potential function to derive the associated characteristic function game, and so the latter will depend on the specific restriction operator $r$ used to define the $r$-potential.

Given any restriction operator $r$, and game $(N, v)$, define the characteristic function $w_{v}^{r}: 2^{N} \rightarrow \mathbb{R}$ as follows:

$$
w_{v}^{r}(N)=v(N), \text { and for all } S \subset N, w_{v}^{r}(S)=v^{-S^{c}, r}(S)
$$

Theorem 1 Let $r$ be a restriction operator satisfying Path Independence. Then for all $(N, v) \in \mathbb{V}$, we have

(i) $P^{r}(N, v)=P\left(N, w_{v}^{r}\right)$.

(ii) $D_{i} P^{r}(N, v)=D_{i} P\left(N, w_{v}^{r}\right)=S h_{i}\left(N, w_{v}^{r}\right)$.

The proof of this and subsequent theorems is in the Appendix.

\subsection{The $r$-Shapley Value}

Let $r$ be any restriction operator satisfying Path Independence. Following Hart and Mas-Colell [7], Theorem 1 allows us to use the $r$-potential to define a value as $S h^{r}$, where

$$
S h_{i}^{r}(N, v) \equiv D_{i} P^{r}(N, v)=P^{r}(N, v)-P^{r}\left(N \backslash\{i\}, v^{-i, r}\right) \text { for all } i \in N
$$

Alternatively,

$$
S h^{r}(N, v)=S h\left(N, w_{v}^{r}\right) .
$$

We will call $S h^{r}$ the $r$-Shapley value.

\subsection{Examples}

The class of restriction operators satisfying Path Independence is very large. We give a few examples to illustrate the richness of this class.

- $\mathbb{P}$-coordinate operator:

Here an a priori partition of the set $\mathbb{N}$ is given and any game is restricted by taking the worth of a coalition when the other players are organized according to

\footnotetext{
${ }^{7}$ See, for instance [4] and [6].
} 
the fixed partition. In other words, any game is restricted by "projecting it onto the coordinate" of this partition. More formally, let $\mathbb{P}$ be a partition of $\mathbb{N}$. Given any $(N, v)$ in $\mathbb{V}$, let for all $i \in N$,

$$
v^{-i, r_{\mathbb{P}}}\left(S ; \pi\left((S \cup\{i\})^{c}\right)\right)=v\left(S ; S^{c} \cap \mathbb{P}\right)
$$

if $(S \cup\{i\})^{c} \cap \mathbb{P}=\pi\left((S \cup\{i\})^{c}\right)$; and $v^{-i, r_{\mathbb{P}}}\left(S ; \pi\left((S \cup\{i\})^{c}\right)\right)=0$ otherwise. We will call $r_{\mathbb{P}}$ the $\mathbb{P}$-coordinate (restriction) operator. It is easy to check that this restriction operator satisfies Path Independence and that for all $(N, v)$ and all $S \subset N$ we have

$$
w_{v}^{r_{\mathbb{P}}}(S)=v\left(S ; S^{c} \cap \mathbb{P}\right) .
$$

The next restriction operator assumes implicitly that a coalition has optimistic expectations about the restricted game.

- Max operator:

It supposes that the worth of the embedded coalition $\left.\left(S ; \pi(S \cup\{i\})^{c}\right)\right)$ in the game $v^{-i}$ equals the maximum worth over all embedded coalitions $(S ; \pi)$ where $\pi \in \pi^{+i}\left((S \cup\{i\})^{c}\right)$.

The $\max$ (restriction) operator is specified below. For all $(N, v) \in \mathbb{V}$ and all $i \in N$, let

$$
v^{-i, \max }\left(S ; \pi\left((S \cup\{i\})^{c}\right)\right)=\max _{\pi \in \pi^{+i}\left((S \cup\{i\})^{c}\right)} v(S ; \pi) .
$$

It is easy to check that the max operator satisfies Path Independence and that for all $(N, v)$ and all $S \subset N$ we have

$$
w_{v}^{\max }(S)=\max _{\pi \in \Pi_{S^{c}}} v(S ; \pi) .
$$

The "dual" of the max operator is the min operator and is obtained by replacing max by $\min$ in the above equalities.

- Weighted average operator:

The weighted average operator $r_{\alpha}$ defines the worth of an embedded coalition $\left(S ; \pi\left((S \cup\{i\})^{c}\right)\right)$ to be a weighted average of the worths of embedded coalitions $\left(S ; \pi^{\prime}\right)$ where $\pi^{\prime} \in \pi^{+i}\left((S \cup\{i\})^{c}\right)$. However, the weights have to be carefully specified in order to satisfy path independence. One possible set of weights is the symmetric weight system, which for every $T \subset \mathbb{N}$ is a vector of weights $\alpha=(\alpha(S ; \pi))_{S \subset \mathbb{N} \backslash T, \pi \in \Pi_{T}}$ such that each weight $\alpha(S ; \pi(T))$ depends only on the distribution of the cardinalities of the elements of $\pi(T)$. For instance, if $T=\{1,2,3\}$, and $S=\{4,5\}$ say, then $\alpha(S ;\{\{1,2\},\{3\}\})=\alpha(S ;\{\{2,3\},\{1\}\})=\alpha(S ;\{\{1,3\},\{2\}\})$ since in each of these three partitions of $T, T$ is partitioned into subsets containing two and one individuals. Then, the operator $r_{\alpha}$ is

$$
v^{-i, r_{\alpha}}\left(S ; \pi\left((S \cup\{i\})^{c}\right)\right)=\sum_{\pi^{\prime} \in \pi^{+i}\left((S \cup\{i\})^{c}\right)} \alpha\left(S ; \pi^{\prime}\right) v\left(S ; \pi^{\prime}\right)
$$


The simplest symmetric weight vector is the one where for each $S \subseteq \mathbb{N}$ and each $\pi(\mathbb{N} \backslash S) \in \Pi_{\mathbb{N} \backslash S}$ we $\operatorname{set}^{8} \alpha(S ; \pi(\mathbb{N} \backslash S))=\frac{1}{\left|\Pi_{\mathbb{N} \backslash S}\right|}$ and for any $S \subset N \subseteq \mathbb{N}$ and $\pi(N \backslash S) \in \Pi_{N \backslash S}$ we set

$$
\alpha(S ; \pi(N \backslash S))=\sum_{\pi(\mathbb{N} \backslash S) \in \Pi_{\mathbb{N} \backslash S}:[(N \backslash S) \cap \pi(\mathbb{N} \backslash S)]=\pi(N \backslash S)} \alpha(S ; \pi(\mathbb{N} \backslash S)) .
$$

For this simplest symmetric weight vector it is easy to verify that for all $(N, v)$ and all $S \subset N$ we have

$$
w_{v}^{r_{\alpha}}(S)=\sum_{\pi(N \backslash S) \in \Pi_{N \backslash S}} \alpha(S ; \pi(N \backslash S)) v(S ; \pi(N \backslash S))=\frac{1}{\mid \Pi_{N \backslash S \mid}} \sum_{\pi(N \backslash S) \in \Pi_{N \backslash S}} v(S ; \pi(N \backslash S))
$$

- Another operator $r^{M}$ is the following.

$$
v^{-i, r^{M}}\left(S ; \pi\left((S \cup\{i\})^{c}\right)\right)=v\left(S ; \pi^{M}\left(S^{c}\right)\right)
$$

where $\pi^{M}\left(S^{c}\right)$ is the partitition in $\pi^{+i}\left(S^{c}\right)$ where $i$ joins the coalition containing $\max \left\{k \mid k \in(S \cup\{i\})^{c}\right\}$, while the rest of the coalitions in $\pi\left((S \cup\{i\})^{c}\right)$ and $\pi\left(S^{c}\right)$ are the same.

- sing operator:

It simply restricts any game $(N, v)$ to $\left(N \backslash\{i\}, v^{-i}\right)$ by assigning to any coalition and any partition the worth of the embedded coalition which results when player $i$ forms a singleton coalition. The sing operator is specified below. For all $(N, v) \in \mathbb{V}$ and all $i \in N$, let

$$
v^{-i, \operatorname{sing}}\left(S ; \pi\left((S \cup\{i\})^{c}\right)\right)=v\left(S ; \pi\left((S \cup\{i\})^{c}\right) \cup\{\{i\}\}\right) .
$$

Clearly, the sing operator satisfies Path Independence. Furthermore, it is easy to verify that for all $(N, v)$ and all $S \subset N$ we have

$$
w_{v}^{\operatorname{sing}}(S)=v\left(S ; \pi^{t}\left(S^{c}\right)\right) .
$$

The sing-Shapley value has played an important role in de Clippel and Serrano [4]. Henceforth we will sometimes follow their terminology and also call it the 'externality free' value.

We will have occasion to use an operator which is related to the sing operator. The $s i n g^{2}$ operator is given by

$$
v^{-i, \text { sing }^{2}}\left(S ; \pi\left((S \cup\{i\})^{c}\right)\right)=\left(v\left(S ; \pi\left((S \cup\{i\})^{c}\right) \cup\{\{i\}\}\right)\right)^{2} .
$$

\footnotetext{
${ }^{8}$ Note that $\mathbb{N}$ is finite.
} 


\section{A Characterisation Theorem}

The examples in the last section illustrate the range of restriction operators which satisfy Path Independence. We now define some other axioms on restriction operators. We then go on to show that these axioms single out a one-parameter class of restriction operators.

First, we define a dummy player. In games without externalities, a dummy player is one whose marginal contribution is zero to all coalitions. By analogy, a dummy player must be one whose marginal contribution is zero to all embedded coalitions. However, this does not uniquely define the concept of a dummy player. One can still define two notions of a dummy player.

Definition 2 Let $(N, v)$ be a game in $\mathbb{V}$.

(i) Player $i \in N$ is a dummy player of type 1 , if for all $S \subseteq N$ containing $i$, and for all partitions $\pi\left(S^{c}\right), v\left(S ; \pi\left(S^{c}\right)\right)-v(S \backslash\{i\} ; \pi)=0$ for all $\pi \in \pi^{+i}\left(S^{c}\right)$.

(ii) Player $i \in N$ is a dummy player of type 2 , if for all $S \subseteq N$ containing $i$, and for all partitions $\pi\left(S^{c}\right), v\left(S ; \pi\left(S^{c}\right)\right)-v\left(S \backslash\{i\} ; \pi\left(S^{c}\right) \cup\{\{i\}\}\right)=0$.

The difference between the two definitions hinges on what player $i$ is supposed to do after she leaves coalition $S$. Player $i$ is said to be a dummy player of type 1 if no assumption is made about what coalition she joins after leaving $S$ - her marginal contribution to $S$ is zero irrespective of which coalition she joins. A player is a type 2 dummy player if her marginal contribution to any embedded coalition is zero when she remains alone after leaving $S$. Clearly, a type 1 dummy player is a type 2 dummy player, though the converse is not true.

Definition 3 A restriction operator $r$ satisfies the Weak Dummy Axiom if for all $(N, v) \in \mathbb{V}$, if player $p$ is a dummy player of type 1 in $(N, v)$, then player $p$ is a dummy player of type 1 in $\left(N \backslash\{i\}, v^{-i}\right)$ for all $i \neq p$.

Definition 4 A restriction operator $r$ satisfies the Strong Dummy Axiom when the following are true for all $(N, v) \in \mathbb{V}$ :

(i) If player $p$ is a dummy player of type 1 in $(N, v)$, then player $p$ is a dummy player of type 1 in $\left(N \backslash\{i\}, v^{-i}\right)$ for all $i \neq p$.

(ii) If player $p$ is a dummy player of type 2 then player $p$ is a dummy player of type 2 in $\left(N \backslash\{i\}, v^{-i}\right)$ for all $i \neq p$.

Both Dummy axioms are designed to capture the idea that a player who is "useless" in the original game should also be useless in all subgames.

The Weak Dummy Axiom does not impose any condition on type 2 dummy players. Hence, the Strong Dummy Axiom implies the Weak Dummy Axiom. We also remind the reader that these are axioms on the restriction operator and not on the 
value. So, the dummy axioms defined above are related but distinct from the dummy axioms on solution concepts which are, for instance, used in the characterization of the Shapley value.

Definition 5 A restriction operator $r$ satisfies Scale Invariance if for all $(N, v),\left(N, v^{\prime}\right) \in$ $\mathbb{V}$ such that $v=a+b v^{\prime}$ for some $a$ and some $b>0,{ }^{9}$ then for all $i \in N, v^{-i}=a+b v^{-i}$.

This axiom requires that if two games on the player set are affine transforms of one another - for instance because individual utility functions undergo affine transformations - then the subgames should also be similarly related.

Definition 6 A restriction operator $r$ satisfies Non-negativity if for all $(N, v) \in \mathbb{V}$, if $v \geq 0^{10}$, then for all $i \in N, v^{-i} \geq 0$.

Another weak axiom is the following.

Definition 7 A restriction operator $r$ satisfies Sign Independence if for all $(N, v),(N, \bar{v}) \in$ $\mathbb{V}$, with $v \geq 0$ and $\bar{v} \leq 0$ such that $v=-\bar{v}$, then for all $i \in N, v^{-i}=-\bar{v}^{-i}$.

Essentially, this axiom says that the process by which subgames are derived in situations where individuals are sharing benefits should be the same as when they are sharing costs.

We first illustrate through examples why some of the possible restriction operators do not satisfy one or more of the axioms defined above.

Properties of simple average (restriction) operator $\left(\bar{r}_{a}\right)$ :

For all $(N, v) \in \mathbb{V}$, for all $i \in N$,

$$
v^{-i, \bar{r}_{a}}\left(S ; \pi\left((S \cup\{i\})^{c}\right)\right)=\frac{1}{K} \sum_{\pi \in \pi^{+i}\left((S \cup\{i\})^{c}\right)} v(S ; \pi)
$$

where $K=\left|\pi^{+i}\left((S \cup\{i\})^{c}\right)\right|$. This operator does not satisfy either Path Independence or the Weak Dummy Axiom. To see why it does not satisfy the Weak Dummy axiom, let $N=\{1,2,3,4\}$ and 4 be a dummy player of type 1 in $(N, v)$. Now,

$v^{-3, \bar{r}_{a}}(\{1\} ;\{2\},\{4\})=1 / 3[v(\{1\} ;\{2,3\},\{4\})+v(\{1\} ;\{2\},\{3,4\})+v(\{1\} ;\{2\},\{4\},\{3\})]$

and

$$
v^{-3, \bar{r}_{a}}(\{1,4\} ;\{2\})=1 / 2[v(\{1,4\} ;\{2,3\})+v(\{1,4\} ;\{2\},\{3\})]
$$

If 4 is a dummy player of type 1 in $v^{-3, \bar{r}_{a}}$, then we must have

$$
v^{-3, \bar{r}_{a}}(\{1\} ;\{2\},\{4\})=v^{-3, \bar{r}_{a}}(\{1,4\} ;\{2\})
$$

\footnotetext{
${ }^{9}$ Abusing notation, $v=a+b v^{\prime}$ means $v\left(S ; \pi\left(S^{c}\right)\right)=a+b v^{\prime}\left(S ; \pi\left(S^{c}\right)\right)$ for all $\emptyset \neq S \subseteq N$ and all $\pi\left(S^{c}\right) \in \Pi_{S^{c}}$.

${ }^{10}$ We use the convention that $x \geq 0$ means $x_{i} \geq 0$ for all $i$ with strict inequality for some coordinate $i$.
} 
Because 4 is a dummy of type 1 in $v, v(\{1,4\} ;\{2,3\})=v(\{1\} ;\{2,3\},\{4\})$ and $v(\{1,4\} ;\{2\},\{3\})=v(\{1\} ;\{2\},\{3,4\})=v(\{1\} ;\{2\},\{4\},\{3\})$. But, then the last equality will hold only if $v(\{1,4\} ;\{2\},\{3\})=v(\{1,4\} ;\{2,3\})$. The fact that 4 is a dummy player in $v$ does not imply the last equality. However, the simple average operator satisfies Scale Invariance and Sign Independence.

Properties of max operator:

For all $(N, v) \in \mathbb{V}$ and all $i \in N$,

$$
v^{-i, \max }\left(S ; \pi\left((S \cup\{i\})^{c}\right)\right)=\max _{\pi \in \pi^{+i}\left((S \cup\{i\})^{c}\right)} v(S ; \pi)
$$

Clearly the max operator fails to satisfy Sign Independence. On the other hand, the max operator satisfies Path Independence, the Weak Dummy Axiom and Scale Invariance.

These examples show that the axioms on restriction operators defined earlier have some "bite". Indeed, the next two theorems provide characterizations of the class of restriction operators which satisfy the two versions of the dummy axiom along with the other axioms.

Theorem 2 Let $r$ be a restriction operator satisfying Path Independence, the Weak Dummy Axiom, Scale Invariance and Sign Independence. Then, there exists $\theta$, such that for all $(N, v) \in \mathbb{V}$ and all $i \in N$,

$v^{-i, r}\left(S ; \pi\left((S \cup\{i\})^{c}\right)\right)=v\left(S ; \pi\left((S \cup\{i\})^{c}\right) \cup\{\{i\}\}\right)+\theta \sum_{\pi \in \pi^{+i}\left((S \cup\{i\})^{c}\right)}\left(v(S ; \pi)-v\left(S ; \pi\left((S \cup\{i\})^{c}\right) \cup\{\{i\}\}\right)\right)$.

Moreover, if the restriction operator satisfies Non-negativity, then $\frac{1}{|\mathbb{N}|-2} \geq \theta \geq 0$.

In words, the class of operators in Theorem 2 does the following: it takes $v\left(S ; \pi\left((S \cup\{i\})^{c}\right) \cup\{\{i\}\}\right)$ as a focal point and then adds to it the differences (or "marginals") of $v(S ; \pi)-v\left(S ; \pi\left((S \cup\{i\})^{c}\right) \cup\{\{i\}\}\right)$ (with $\left.\pi \in \pi^{+i}\left((S \cup\{i\})^{c}\right)\right)$. The parameter $\theta$ measures the importance of these "marginals" on the restriction operator. It is easy to see that these restriction operators satisfy all axioms of Theorem 2 .

We may rewrite (1) and obtain

$v^{-i, r}\left(S ; \pi\left((S \cup\{i\})^{c}\right)\right)=\theta \sum_{\pi \in \pi^{+i}\left((S \cup\{i\})^{c}\right)} v(S ; \pi)+(1-t \theta) v\left(S ; \pi\left((S \cup\{i\})^{c}\right) \cup\{\{i\}\}\right)$

where $t=\left|\pi^{+i}\left((S \cup\{i\})^{c}\right)\right|^{11}$ This means that $r_{i, S, \pi\left(S^{c}\right)}^{N}$ is a linear combination of the simple average operator and the sing operator.

However, if the Weak Dummy Axiom is replaced by its stronger counterpart, then we are left only with the sing operator. This is the content of the next theorem.

\footnotetext{
${ }^{11}$ Note that $\left.\pi\left((S \cup\{i\})^{c}\right) \cup\{i\}\right)$ is an element of $\pi^{+i}\left((S \cup\{i\})^{c}\right)$. So, the total weight on $v(S ; \pi(S \cup$ $\left.\left.\left.\{i\})^{c}\right)\right) \cup\{i\}\right)$ is $(1-(t-1) \theta)$.
} 
Theorem 3 Let $r$ be a restriction operator satisfying Path Independence, the Strong Dummy Axiom, Scale Invariance and Sign Independence. Then $r=\operatorname{sing}$.

We can obtain a slightly different version of Theorem 2 by replacing Sign Independence and Non-negativity with Monotonicity, a condition which is defined below.

Definition 8 Let $(N, v),\left(N, v^{\prime}\right) \in \mathbb{V}$ be such that for some $S \subset N, \pi\left(S^{c}\right) \in \Pi_{S^{c}}$, $v\left(S ; \pi\left(S^{c}\right)\right)>v^{\prime}\left(S ; \pi\left(S^{c}\right)\right)$ while $v\left(T ; \pi\left(T^{c}\right)\right)=v^{\prime}\left(T ; \pi\left(T^{c}\right)\right)$ for all other embedded coalitions $\left(T ; \pi\left(T^{c}\right)\right)$. A restriction operator $r$ satisfies Monotonicity if for all $i \in S^{c}$ and for all $\pi\left((S \cup\{i\})^{c}\right) \in \Pi_{(S \cup\{i\})^{c}}, v^{-i}\left(S ; \pi\left((S \cup\{i\})^{c}\right)\right) \geq v^{\prime-i}\left(S ; \pi\left((S \cup\{i\})^{c}\right)\right)$.

This axiom implies that $r_{i, S, \pi\left((S \cup\{i\})^{c}\right)}^{N}$ is monotonic for all $i, S$ and $\pi\left((S \cup\{i\})^{c}\right)$.

Theorem 4 Let $r$ be a restriction operator satisfying Path Independence, the Weak Dummy Axiom, Scale Invariance, and Monotonicity. Then, $r$ must be one of the following. For all $(N, v) \in \mathbb{V}$ and all $i \in N$,

- There exists $\theta$, such that

$$
\begin{aligned}
& v^{-i, r}\left(S ; \pi\left((S \cup\{i\})^{c}\right)\right)=\theta \sum_{\pi \in \pi^{+i}\left((S \cup\{i\})^{c}\right)} v(S ; \pi)+(1-t \theta) v\left(S ; \pi\left((S \cup\{i\})^{c}\right) \cup\{\{i\}\}\right) \\
& \text { where } t=\left|\pi^{+i}\left((S \cup\{i\})^{c}\right)\right| \text { and } \frac{1}{|\mathbb{N}|-2} \geq \theta \geq 0 . \\
& \text { - } v^{-i, r}\left(S ; \pi\left((S \cup\{i\})^{c}\right)\right)=\max _{\pi \in \pi^{+i}\left((S \cup\{i\})^{c}\right)} v(S ; \pi) \\
& \text { - } v^{-i, r}\left(S ; \pi\left((S \cup\{i\})^{c}\right)\right)=\min _{\pi \in \pi^{+i}\left((S \cup\{i\})^{c}\right)} v(S ; \pi)
\end{aligned}
$$

We have mentioned earlier that the max and min operators do not satisfy Sign Independence. Essentially, this theorem shows that if Sign Independence is not imposed on the restriction operator, then the only additional rules allowed are these two operators. ${ }^{12}$

In order to prove the independence of the axioms in Theorems 2, 3 and 4, we need to introduce a few additional operators.

First, we give an example of an operator which violates Path Independence, but satisfies the other axioms in Theorem 3.

For each $i \in N$, let $\sigma_{i}$ denote individual $i$ 's strict preference ordering over $\mathbb{N} \backslash\{i\}$, and $\sigma=\left(\sigma_{i}\right)_{i \in \mathbb{N}}$. Then,

$$
v^{-i, r^{\sigma}}\left(S ; \pi\left((S \cup\{i\})^{c}\right)\right)=v\left(S ; \pi\left(S^{c}\right)\right)
$$

where $i$ joins the coalition which contains the $\sigma_{i}$-maximal individual element in the set of individuals who are not dummies of type 1 and who are in $\left.(S \cup\{i\})^{c}\right)$. If all the players in $\left.(S \cup\{i\})^{c}\right)$ are dummy players of type 1, then $i$ remains alone.

\footnotetext{
${ }^{12}$ The proof of this theorem is quite long and involved. We have not reported it in the Appendix, but it is contained in the Supplement of this paper.
} 
Finally, the following operator violates Monotonicity, but satisfies the other axioms in Theorem 4. Choose $\theta<0$ and define

$v^{-i, r^{\theta^{*}}}\left(S ; \pi\left((S \cup\{i\})^{c}\right)\right)=\theta \sum_{\pi \in \pi^{+i}\left((S \cup\{i\})^{c}\right)} v(S ; \pi)+(1-t \theta) v\left(S ; \pi\left((S \cup\{i\})^{c}\right) \cup\{\{i\}\}\right)$

where $t=\left|\pi^{+i}\left((S \cup\{i\})^{c}\right)\right|{ }^{13}$

Table 1 illustrates the independence of the axioms in Theorem 2, Theorem 3 and Theorem 4. The max operator satisfies all axioms except Sign Independence. The operator $r^{M}$ satisfies all axioms except the Weak Dummy axiom, while $r^{\sigma}$ violates Path Independence, but satisfies the other axioms. The operator $\operatorname{sing}^{2}$ does not satisfy Scale Invariance, but satisfies the other axioms, while $r^{\theta^{*}}$ completes the demonstration that the axioms in Theorem 4 are independent.

\footnotetext{
${ }^{13} \mathrm{~A}$ comparison with the class of operators characterized in Theorem 2 shows that $r^{\theta *}$ satisfies all the axioms in that theorem except Sign Independence. Also, it does not satisfy Non-negativeness.
} 
Table 1: Properties of Various Operators

\begin{tabular}{|c|c|c|c|c|c|c|}
\hline Operators & Path Ind & Strong Dummy & Weak Dummy & Scale Inv & Sign Ind & Mon \\
\hline $\bar{r}_{a}$ & $\mathrm{~N}$ & $\mathrm{~N}$ & $\mathrm{~N}$ & $\mathrm{Y}$ & $\mathrm{Y}$ & $\mathrm{Y}$ \\
\hline$r_{\alpha}$ & $\mathrm{Y}$ & $\mathrm{N}$ & $\mathrm{N}$ & $\mathrm{Y}$ & $\mathrm{Y}$ & $\mathrm{Y}$ \\
\hline Max & $\mathrm{Y}$ & $\mathrm{Y}$ & $\mathrm{Y}$ & $\mathrm{Y}$ & $\mathrm{N}$ & $\mathrm{Y}$ \\
\hline Min & $\mathrm{Y}$ & $\mathrm{N}$ & $\mathrm{Y}$ & $\mathrm{Y}$ & $\mathrm{N}$ & $\mathrm{Y}$ \\
\hline$r_{\mathbb{P}}$ & $\mathrm{Y}$ & $\mathrm{N}$ & $\mathrm{N}$ & $\mathrm{N}$ & $\mathrm{Y}$ & $\mathrm{Y}$ \\
\hline $\operatorname{sing}$ & $\mathrm{Y}$ & $\mathrm{Y}$ & $\mathrm{Y}$ & $\mathrm{Y}$ & $\mathrm{Y}$ & $\mathrm{Y}$ \\
\hline$r^{M}$ & $\mathrm{Y}$ & $\mathrm{N}$ & $\mathrm{N}$ & $\mathrm{Y}$ & $\mathrm{Y}$ & $\mathrm{Y}$ \\
\hline$r^{\sigma_{i}}$ & $\mathrm{~N}$ & $\mathrm{Y}$ & $\mathrm{Y}$ & $\mathrm{Y}$ & $\mathrm{Y}$ & $\mathrm{Y}$ \\
\hline $\operatorname{sing}^{2}$ & $\mathrm{Y}$ & $\mathrm{Y}$ & $\mathrm{Y}$ & $\mathrm{N}$ & $\mathrm{Y}$ & $\mathrm{Y}$ \\
\hline$r^{\theta^{*}}$ & $\mathrm{Y}$ & $\mathrm{N}$ & $\mathrm{Y}$ & $\mathrm{Y}$ & $\mathrm{Y}$ & $\mathrm{N}$ \\
\hline
\end{tabular}

Note: "Y" denotes that the property is satisfied, while "N" denotes that the property is not satisfied by the property in question.

\section{The Shapley Axioms}

We now show that the class of values resulting from the restriction operators characterised in Theorem 2 satisfy all the natural extensions of Shapley's classic axioms to games in partition function form.

We start by defining these axioms.

Definition 9 Let $\varphi$ be a solution on $\mathbb{V}$. Then, $\varphi$ satisfies

(i) Linearity: if

(a) For all $(N, v),\left(N, v^{\prime}\right) \in \mathbb{V}, \varphi\left(N, v+v^{\prime}\right)=\varphi(N, v)+\varphi\left(N, v^{\prime}\right)$; and

(b) For any scalar $\lambda \in \mathbb{R}$ and any game $(N, v) \in \mathbb{V}, \varphi(N, \lambda v)=\lambda \varphi(N, v)$.

(ii) Symmetry: if for any permutation $\sigma$ of $N, \varphi(N, \sigma v)=\sigma \varphi(N, v)$.

(iii) Efficiency: if for all $(N, v) \in \mathbb{V}, \sum_{i \in N} \varphi_{i}(N, v)=v(N)$.

(iv) Weak Dummy Property: if for all $(N, v) \in \mathbb{V}, \varphi_{i}(N, v)=0$ if $i$ is a type 1 dummy player.

(v) Strong Dummy Property: if for all $(N, v) \in \mathbb{V}, \varphi_{i}(N, v)=0$ if $i$ is a type 2 dummy player. ${ }^{14}$

\footnotetext{
${ }^{14}$ Notice that these Dummy properties are restrictions on the solution concept and are distinct from the Dummy axioms which were imposed on the restriction operators.
} 
Suppose now that the restriction operator $r$ satisfies Path Independence, the Weak Dummy Axiom, Scale Invariance, Sign Independence, and Non-negativity. Then, $r$ satisfies (1) in Theorem 2. In addition to the fact that $r$ is described by (1), we use from Theorem 1 the fact that $\operatorname{Sh}^{r}(N, v)=S h\left(N, w_{v}^{r}\right)$.

Consider first the property of Linearity. Take any two games $(N, v)$ and $\left(N, v^{\prime}\right)$. Then, it is easy to check from (1), that

$$
w_{v+v^{\prime}}^{r}=w_{v}^{r}+w_{v^{\prime}}^{r}
$$

and for any scalar $\lambda$,

$$
w_{\lambda v}^{r}=\lambda w_{v}^{r} .
$$

Moreover, since the Shapley value on $\mathbb{W}$ satisfies Linearity, it follows that

$$
S h^{r}\left(N, v+v^{\prime}\right)=S h\left(N, w_{v}^{r}\right)+S h\left(N, w_{v^{\prime}}^{r}\right)=S h^{r}(N, v)+S h^{r}\left(N, v^{\prime}\right)
$$

and

$$
S h^{r}(N, \lambda v)=S h\left(N, w_{\lambda v}^{r}\right)=S h\left(N, \lambda w_{v}^{r}\right)=\lambda S h\left(N, w_{v}^{r}\right)=\lambda S h^{r}(N, v) .
$$

These are sufficient to show that $S h^{r}$ satisfies Linearity on $\mathbb{V}$.

Now, consider any permutation $\sigma$ of $N$. Then,

$$
w_{\sigma v}^{r}=\sigma w_{v}^{r}
$$

Since the Shapley value satisfies Symmetry on $\mathbb{W}$, this last equality shows that the property of Symmetry can be extended to $\mathbb{V}$. It is also obvious that $S h^{r}$ satisfies Efficiency.

For the Weak Dummy Property, choose any $(N, v) \in \mathbb{V}$ and suppose $i \in N$ is a dummy player of type 1 in $v$. Choose any $S$ containing $i$. Now, from repeated application of the Weak Dummy Axiom on $r, i$ is a type 1 dummy player in $v^{-S^{c}, r}$. Hence,

$$
v^{-S^{c}, r}(S)=v^{-S^{c}, r}(S \backslash\{i\} ;\{i\})
$$

Also,

$$
w_{v}^{r}(S)=v^{-S^{c}, r}(S)=v^{-S^{c}, r}(S \backslash\{i\} ;\{i\})=v^{-(S \cup\{i\})^{c}, r}(S \backslash\{i\})=w_{v}^{r}(S \backslash\{i\})
$$

This shows that $i$ is a dummy player of type 1 in $w_{v}^{r}$ and by the Weak Dummy Property of the Shapley value on TU games, $S h_{i}^{r}(N, v)=0$.

A similar analysis applies to the Strong Dummy Property and the Strong Dummy Axiom.

Of course, there are other solution concepts outside the class $\left\{S h^{r}\right\}$ where $r$ satisfies the axioms specified in Theorem 2 which also satisfy the Shapley axioms for partition function games. ${ }^{15}$

\footnotetext{
${ }^{15}$ See for instance [1], [3], [5], [6], [8], [10] and [13].
} 


\section{Consistency}

In the last section we have compared our results with the classical axiomatization of the Shapley value. Hart and Mas-Colell [7] provided an alternative axiomatic derivation of the Shapley value in characteristic function games through the use of an internal consistency condition which has come to be called a "reduced game" consistency property. ${ }^{16}$ The intuitive content of this concept of consistency is the following. Suppose $\varphi$ is a solution concept, and that a group of players can be "bought off" by paying them according to $\varphi$. These players do not actually leave the game, but can be persuaded to cooperate with any coalition provided they are paid according to $\varphi$. This then precipitates a reduced game on the complementary player set $S^{c}$, and $\varphi$ is said to satisfy (reduced game) consistency if it prescribes the same payoffs to players in $S^{c}$ in both the reduced game as well as the original game for the grand coalition.

There are different definitions of reduced games, each corresponding to a different interpretation of what it means for the coalition $S$ to be paid according to the solution concept $\varphi$. Below we define the natural extension of the reduced game formulated by Hart and Mas-Colell to partition function form games. As one might imagine, each restriction operator gives rise to one such reduced game and to a corresponding notion of consistency.

It is worth pointing out here that a "reduced" game is different from a subgame. This difference is more transparent when the original game is without externalities. As we have pointed out earlier, the subgame on player set $S$ is simply the projection of the original game to $S$ and subsets of $S$. That is, there is no ambiguity about the subgame. However, there are different versions of a reduced game even in this case.

Definition 10 Fix a restriction operator $r$ satisfying Path Independence. Let $\varphi$ be a value and $(N, v) \in \mathbb{V}$. For any $S \subset N$, the reduced game $\left(S, v_{S}^{\varphi, r}\right)$ is defined as follows. For all $R \subseteq S$ and all $\pi(S \backslash R) \in \Pi_{S \backslash R}$,

$$
v_{S}^{\varphi, r}(R ; \pi(S \backslash R))=v\left(R \cup S^{c} ; \pi(S \backslash R)\right)-\sum_{k \in S^{c}} \varphi_{k}\left(R \cup S^{c}, v^{-(S \backslash R), r}\right)
$$

So, the Hart and Mas-Colell reduced game on the player set $S$ specifies that if players in $S^{c}$ join forces with some $R \subseteq S$, then they are paid what they would obtain (according to $\varphi$ ) in the subgame restricted to the player set $S^{c} \cup R$. Of course, this subgame depends on the specific restriction operator. ${ }^{17}$

This reduced game leads to the following definition of consistency.

Definition 11 A value $\varphi$ is r-consistent iff for all $(N, v) \in \mathbb{V}$, all $S \subset N$ and all $i \in S$, we have

$$
\varphi_{i}(N, v)=\varphi_{i}\left(S, v_{S}^{\varphi, r}\right)
$$

\footnotetext{
${ }^{16}$ Different versions of the reduced game property have proved very useful in the characterization of a variety of cooperative solution concepts. See for instance [1], [3], [7], [9], [10], and [14].

${ }^{17}$ Notice that the Hart and Mas-Colell reduced game for TU games is obtained from Definition 10 by dropping $\pi(S \backslash R)$ from it.
} 
Hart and Mas-Colell [7] showed that on the class of games $\mathbb{W}$, the Shapley value is the only solution satisfying consistency and the "standard property" which requires that on two-person games, the solution splits the gains from cooperation equally between the two players.

Definition 12 A value $\varphi$ satisfies the standard property on two-person games if for all $(\{i, j\}, v) \in \mathbb{V}, \varphi_{i}(\{i, j\}, v)=v(\{i\})+\frac{1}{2}[v(\{i, j\})-v(\{i\})-v(\{j\})]^{18}$

In this section, we prove an analogous result for partition function games. For this uniqueness result we need two very mild additional axioms on restriction operators.

Definition 13 A restriction operator $r$ satisfies Translation Invariance if for all player sets $N$, all $i \in N$, all $S \subseteq N \backslash\{i\}$, and all $\pi\left((S \cup\{i\})^{c}\right)$, and any real numbers $x_{1}, \ldots, x_{k}$ and $c$,

$$
r_{i, S, \pi\left((S \cup\{i\})^{c}\right)}^{N}\left(x_{1}-c, \ldots, x_{k}-c\right)=r_{i, S, \pi\left((S \cup\{i\})^{c}\right)}^{N}\left(x_{1}, \ldots, x_{k}\right)-c .
$$

Translation Invariance simply says that if the original worths are translated by some constant, then in the restricted game the worth should also be translated by the same constant. Note that Scale Invariance implies Translation Invariance.

Definition 14 A restriction operator satisfies Limited Independence if for all $N_{1} \subseteq$ $N_{2} \subseteq \mathbb{N}$ and $i \in N_{1}$,

$$
r_{i, S, \pi(T)}^{N_{1}}=r_{i, S \cup\left(N_{2}-N_{1}\right), \pi(T)}^{N_{2}}
$$

when $S \subseteq\left(N_{1}-\{i\}\right)$ and any $T=N_{1}-(S \cup\{i\}) .{ }^{19}$

Limited independence identifies a situation where the restriction operator does not vary with the original player set. To some extent, this is a technical condition and has no obvious intuitive appeal. However, we will show in Lemma 5 in the Appendix that that this condition is actually an implication of Path Independence, Scale Invariance, Sign Independence and the Weak Dummy Axiom.

We are now ready for the main theorem of this section.

Theorem 5 Let $r$ be a restriction operator satisfying Path Independence, Limited Independence and Translation Invariance. Then, a value $\varphi$ satisfies $r$-consistency and the standard property on two-person games if and only if $\varphi \equiv S h^{r}$.

The following corollaries follow from Theorem 5 because the classes of operators described in the corollaries either satisfy the three conditions imposed in the theorem or the proof of the theorem can be easily adapted to the specific case.

Corollary 1 A value $\varphi$ satisfies max-consistency and the standard property on twoperson games if and only if $\varphi \equiv S h^{\max }$.

\footnotetext{
${ }^{18}$ Abusing notation, for two player games we write $v(\{i\})$ instead of $v(\{i\} ;\{j\})$.

${ }^{19}$ This means that for any real numbers $x_{1}, \ldots, x_{k}$ (where $k=|\pi(T)|+1$ ) we have $r_{i, S, \pi(T)}^{N_{1}}\left(x_{1}, \ldots, x_{k}\right)=r_{i, S \cup\left(N_{2}-N_{1}\right), \pi(T)}^{N_{2}}\left(x_{1}, \ldots, x_{k}\right)$.
} 
Corollary 2 A value $\varphi$ satisfies sing-consistency and the standard property on twoperson games if and only if $\varphi \equiv S h^{\text {sing }}$.

Corollary 3 Let $\alpha$ be a symmetric weight system. A value $\varphi$ satisfies $r_{\alpha}$-consistency and the standard property on two-person games if and only if $\varphi \equiv S h^{r_{\alpha}}$.

Although the $\mathbb{P}$-coordinate operator does not satisfy Translation Invariance, we obtain from Theorem 5 and its proof a similar corollary for it.

Corollary 4 Let $\mathbb{P}$ be a partition of the set $\mathbb{N}$. A value $\varphi$ satisfies $r_{\mathbb{P}}$-consistency and the standard property on two-person games if and only if $\varphi \equiv S h^{r_{\mathbb{P}}}$.

Since Scale Invariance implies Translation Invariance, the corollary below follows from Theorem 5 and Lemma 5.

Corollary 5 Let $r$ be a restriction operator satisfying Path Independence, the Weak Dummy Axiom, Scale Invariance and Non-negativity. Then, a value $\varphi$ satisfies $r$ consistency and the standard property on two-person games if and only if $\varphi \equiv S h^{r}$.

\section{Concluding Remarks}

In this paper, we have used the tool of restriction operators to define subgames of partition function games. Once subgames are well defined, it is possible to use the potential approach à la Hart and Mas-Colell to construct values for partition function games. We show that if the restriction operator satisfies the basic assumption of Path Independence, then it is possible to relate the potential of any partition function game to that of a specific characteristic game. One can then define the value of the partition function game to coincide with the Shapley value of the derived characteristic function game. We also show that a large class of such values are characterised by a natural extension of the reduced game consistency property of Hart and Mas-Colell and standardness. Finally, we adopt an axiomatic approach to narrow down the class of permissible restriction operators. In this characterisation we use Path Independence, Scale Invariance and the Weak Dummy Axiom. The last two properties are parallel to the corresponding properties used by Shapley's [15] classic characterisation.

We show that only a strong version of the Dummy player axiom yields a unique value. This is in common with several recent contributions which possess the same characteristic. That is, these papers also show that a large class of values satisfies a set of basic axioms, and that a unique value is generated only if an additional axiom is added to the basic set.

Myerson [10] was the first to derive a value concept for games in partition form. He imposed a strong "carrier" axiom to derive a unique value. De Clippel and Serrano [5] point out that Myerson's value sometimes yields unintuitive predictions.

Macho-Stadler et al. [8] follow a two-stage procedure which they call the "average approach". The average approach too generates a characteristic function game 
from the partition function game by specifying that the worth of each coalition $S$ is the weighted average of the worths of embedded coalitions $(S ; \pi)$ where $\pi$ is some partition of the complementary coalition. The Symmetry and Dummy axioms of Shapley impose some restrictions on the permissible sets of weights. However, these permissible weights yield a large class of weighting schemes and hence result in a large class of Shapley values. An additional axiom is then used to single out a specific set of weights - this obviously yields a unique characteristic function game for each partition function game. Furthermore, the value characterized by Albizuri et al. [1] belongs to the values proposed by Macho-Stadler et al. [8] - it is identical with the Shapley value associated with the simple average operator.

Bolger [3] uses the additivity axiom, which is weaker than Linearity. But, he derives a unique value by imposing an additional axiom of expected marginality on the class of simple games. It turns out that Bolger's value cannot be derived from the "average" approach.

De Clippel and Serrano [5] follow Young's [19] approach for characteristic function games. In particular, their basic axiom requires that if between two partition function games $v$ and $v^{\prime}$, player $i$ 's marginal contributions vector to every embedded coalition is higher in $v$ than in $v^{\prime}$, then player $i$ 's value in $v$ should also be at least as high as in $v^{\prime}$. However, this marginality principle too does not have enough bite to single out a unique value. De Clippel and Serrano then define player $i$ 's intrinsic marginal contribution to an embedded coalition $(S ; \pi)$ to be the difference to $S$ created if $i$ leaves $S$ and remains alone instead of joining any element of the partition $\pi$. They then show that only $S h^{\text {sing }}$ satisfies the stronger marginality principle. ${ }^{2021}$

Our approach includes all these extensions of the Shapley value from characteristic function games to games in partition functions form. As our examples in Section 3.3 show, the $r$-Shapley values allow for taking simple or weighted averages, capturing pessimistic or optimistic expectations and the externality free value. The restriction operators characterised in Theorem 2 are for each $N, i, S$, and $\pi\left(S^{c}\right)$ a linear combination of the simple average approach and the externality free value. This captures simultaneously the two ideas of the simple average approach and of any agent remaining alone once he leaves a coalition.

We conclude with some examples to illustrate the difference between our values and those of de Clippel and Serrano [5] and Macho-Stadler et al [8].

Example 1 Let $N=\{1,2,3\}, v(N)=v(\{1, j\} ;\{k\})=v(\{1\} ;\{2\},\{3\}\})=1$, $v(S ; \pi)=0$ otherwise.

Players 2 and 3 are Type 2-dummy players, and so $S h^{\operatorname{sing}}(v)=(1,0,0)$.

But, notice that players 2 and 3 are not powerless because they can drive 1's worth down to 0 by joining forces. This power should be reflected in "some" positive payoff.

\footnotetext{
${ }^{20}$ Fujinaki [6] is a variant of this marginality approach in that he uses different exogenous weighting schemes to aggregate the different possibilities when a player $i$ leaves a coalition.

${ }^{21}$ Pham Do and Norde [13] also characterize the externality free value using the Strong Dummy Property for values.
} 
In Theorem 2 for $\theta=1 / 2$ we have $S h^{r}(v)=(5 / 6,1 / 12,1 / 12)$. This is also the unique value selected by Macho-Stadler et al. [8] for this game. In fact, when $|N|=3$, the unique value selected in [8] coincides always with $S h^{r}$ for $\theta=1 / 2$ in Theorem 2.

We now introduce a mild axiom on a solution concept in order to illustrate a crucial difference between the values of $S h^{r}$ corresponding to restriction operators derived from equation(1) for $\theta>0$ and $S h^{\operatorname{sing}}$ which corresponds to $\theta=0$.

Definition 15 A solution $\varphi$ on $\mathbb{V}$ satisfies Strict Ranking if for all $i, j \in N, \varphi_{i}(v)>$ $\varphi_{j}(v)$ whenever the following is true.

$\forall S \subset N \backslash\{i, j\}, v(S \cup\{i\} ; \pi) \geq v\left(S \cup\{j\} ; \pi^{\prime}\right) \forall \pi \in \Pi_{(S \cup\{i\})^{c}}, \pi^{\prime} \in \Pi_{(S \cup\{j\})^{c}}$, with strict inequality for some $\pi, \pi^{\prime}$

This condition simply states that if $i$ is in an obvious sense more desirable than $j$, then $i$ should be allocated more than $j$ by the solution concept.

Now, consider the following example.

Example 2 Let $N=\{1,2,3\} ; v(N)=1=v(\{1\} ;\{2,3\})$, for $i, j \in\{2,3\}, v(\{j\} ;\{1, i\})=$ $-K$ where $K$ is a very large number, and $v(S ; \pi)=0$ otherwise.

Each player's intrinsic marginal contribution vector is identical, and so $S h^{\operatorname{sing}}(v)=$ $(1 / 3,1 / 3,1 / 3)$. But, clearly the game is not symmetric, and player 1 should get more than players 2 and 3. Since this is also required by Strict Ranking, the example shows that $S h^{\text {sing }}$ violates this condition. However, Strict ranking is satisfied by all $S h^{r}$ if $r$ corresponds to $\theta>0$ in equation (1). For $\theta=1 / 2, S h^{r}$ and the unique value in [8] specify the payoff vector $(1 / 2+K / 6,1 / 4-K / 12,1 / 4-K / 12)$.

Example 3 This example is due to de Clippel and Serrano [5]: Let $|N|=101$, $v(N)=v\left(\{1\} ; \pi^{t}(N \backslash\{1\})\right)=1$, and $v(S ; \pi)=0$ otherwise.

In this example, 1 should clearly get more than the other players. But, how much more? Macho-Stadler et al.'s value is very close to equal split, with 1 getting slightly more. On the other hand, $S h_{1}^{\text {sing }}=2 / 101$, and $S h_{j}^{\text {sing }}=(99 / 10100)$ for other $j$. So, 1 gets just over double what the other players get. In Theorem $2, S h^{r}$ will vary between these two extremes as $\theta$ changes from 0 to $1 / 2$.

The three examples above illustrate the flexibility of $S h^{r}$ - while $\theta=0$ sometimes leads to unintuitive predictions, higher values of $\theta$ result in values which could be more appealing. 


\section{REFERENCES}

1. M.J. Albizuri, J. Arin, J. Rubio, An Axiom System for a value for games in partition function form, Int. Game Theory Rev. 7 (2005), 63-73.

2. R.J. Aumann, M. Maschler, Game-theoretic analysis of a bankruptcy problem from the Talmud, J. Econ. Theory 36 (1985), 195-213.

3. E.M. Bolger, A set of axioms for a value for partition function games, Int. J. Game Theory 38 (1989), 37-44.

4. M. Davis, M. Maschler, The kernel of a cooperative Game, Naval Research Logistics Quarterly 12 (1965), 223-259.

5. G. de Clippel, R. Serrano, Marginal contributions and externalities in the value, Econometrica 76 (2008), 1413-1436.

6. Y. Fujinaka, On the marginality principle in partition function games, mimeo (2004), Graduate School of Economics, Kobe University.

7. S. Hart, A. Mas-Colell, Potential, value and consistency, Econometrica 57 (1989), 589-614.

8. I. Macho-Stadler, D. Perez-Castrillo, D.Wettstein, Sharing the surplus: An extension of the Shapley Value for environments with externalities, J. Econ. Theory 135 (2007), 339-356.

9. H. Moulin, The separability axiom and equal-sharing methods, J. Econ. Theory 36 (1985), 120-148.

10. R. Myerson, Values of games in partition function form, Int. J. Game Theory 6 (1977), 23-31.

11. B. Peleg, An axiomatization of the core of cooperative games without side payments, J. Math. Econ. 14 (1985), 203-214.

12. B. Peleg, An axiomatization of the core of cooperative games without side payments, Int. J. Game Theory 15 (1986), 187-200.

13. K.H. Pham Do, H. Norde, The Shapley value for partition function form games, Int. Game Theory Rev. 9 (2007), 353-360.

14. D. Ray, A game-theoretic perspective on coalition formation, Oxford University Press, Oxford, 2007.

15. L.S. Shapley, (1953), A value for $n$-person games, in: A.W.Tucker and R.D.Luce (Eds.), Contributions to the Theory of Games II, Princeton University Press, 1953, pp. 307-317. 
16. A.I. Sobolev, The characterization of optimality principles in cooperative games by functional equations, in: N.N. Vorobev (Editor), Mathematical Methods in Social Sciences, Vilnius, 1975, pp. 94-151.

17. W. Thomson, Monotonicity, stability and egalitarianism, Math. Soc. Sci. 8 (1984), 15-28.

18. R.M. Thrall, W.F. Lucas, n-person games in partition function form, Naval Research Logistics Quarterly 10 (1963), 281-298.

19. H.P. Young, Monotonic solutions of cooperative games, Int. J. Game Theory 14 (1985), 65-72. 


\begin{abstract}
APPENDIX
In the Appendix we often use the following (simplified) notation. Whenever there is no scope for confusion, we will write a set $S=\{i, j, k\}$ as $i j k$, etc. For any set $S$ and $i \in S^{c}$, we write $S+i$ to denote the set $S \cup\{i\}$, and $S-i$ to denote the set $S-\{i\}$. Similarly, $S+i j$ denotes the set $S \cup\{i, j\}$. We will also write a partition $\{\{i j\},\{k l\}\}$ as $\{i j, k l\}$, etc. That is elements in a partition will be separated by a "comma".
\end{abstract}

\title{
Proof of Theorem 1:
}

We only need to prove (i) since (ii) is an immediate consequence of (i) and Theorem 1 of Hart and Mas-Colell [7]. We prove (i) by induction on $|N|$.

If $|N|=1$, say $N=\{i\}$, then by definition of $w_{v}^{r}, w_{v}^{r}(i)=v(i)$. Hence, (i) is true for one-person games since by definition of $w_{v}^{r}, P\left(i, w_{v}^{r}\right)=w_{v}^{r}(i)=v(i)$. Also, $P^{r}(i, v)=v(i)$ from the definition of a potential.

Let $N$ be a player set and suppose by induction that (i) is true for all player sets containing fewer than $|N|$ players. Let $i \in N$. We first show that for all $S \subseteq N-i$

$$
w_{v}^{r}(S)=w_{v^{-i, r}}^{r}(S) .
$$

where of course $v^{-i, r}$ is the partition function induced by $r$ on player set $N-i$. Note that from Path Independence,

$$
v^{-S^{c}}=\left(v^{-i}\right)^{-\left(S^{c}-i\right)}
$$

where we have dropped reference to the restriction operator for notational simplicity. Hence, for all $S \subseteq N-i$, we have

$$
w_{v}^{r}(S)=v^{-S^{c}}(S)=\left(v^{-i}\right)^{-\left(S^{c}-i\right)}(S)=w_{v^{-i, r}}^{r}(S)
$$

where the first and the third equality follow from the definition of $w$, and the second equality follows from (3). Hence, (2) is true.

Let $\left.w_{v}^{r}\right|_{N-i}$ denote the subgame of $w_{v}^{r}$ on the player set $N-i$. Now we obtain

$$
\begin{aligned}
P^{r}(N, v) & =\frac{1}{|N|}\left(v(N)+\sum_{i \in N} P^{r}\left(N-i, v^{-i, r}\right)\right) \\
& =\frac{1}{|N|}\left(w_{v}^{r}(N)+\sum_{i \in N} P\left(N-i, w_{v^{-i, r}}^{r}\right)\right) \\
& \left.=\frac{1}{|N|}\left(w_{v}^{r}(N)+\sum_{i \in N} P\left(N-i,\left.w_{v}^{r}\right|_{N-i}\right)\right)\right) \\
& =P\left(N, w_{v}^{r}\right)
\end{aligned}
$$

where the first equality follows from the definition of an $r$-potential, the second from our induction hypothesis that (i) is true for all player sets containing fewer than $|N|$ 
players, the third from (2), and the fourth from the definition of the characteristic function potential.

The following lemmata are important for the proof of Theorem 2. Lemma 1 simply connects restriction operator of an arbitrary partition to that of a trivial partition. Suppose that we are interested in the subgame $v^{-i}$. Consider any $S$, and any partition of the complementary coalition, $\pi\left((S+i)^{c}\right)=\left\{S_{1}, \ldots, S_{K}\right\}$. Consider another configuration where one agent from each of $S_{1}, \ldots, S_{K}$ stays back, while the rest join $S$. Lemma 1 shows that the restriction operator $r_{i, S, \pi\left((S+i)^{c}\right)}^{N}$ is identical to $r_{i, S \cup N^{\prime}, \pi^{t}\left(\left(\left\{j_{1}, \ldots, j_{K}\right\}\right)\right)}^{N}$, where $j_{k} \in S_{k}$ for all $k$ and $N^{\prime}=\cup_{k=1}^{K}\left(S_{k}-j_{k}\right)$. This will allow us to prove the main theorem only for trivial partitions, which can then be extended (for an arbitrary partition) through Lemma 1.

Lemma 1 Let $r$ be a restriction operator satisfying Path Independence and the Weak Dummy Axiom. Let $N \subseteq \mathbb{N}$ and $i \in N$. Choose any $S \subseteq(N-i)$ and any partition $\pi\left((S+i)^{c}\right)=\left\{S_{1}, \ldots, S_{K}\right\}$. For any choice of $j_{k} \in S_{k}, k=1, \ldots K$, define $N^{\prime}=$ $\cup_{k=1}^{K}\left(S_{k}-j_{k}\right)$. Then $r_{i, S, \pi\left((S+i)^{c}\right)}^{N}=r_{i, S \cup N^{\prime}, \pi^{t}\left(\left\{j_{1}, \ldots, j_{K}\right\}\right)}^{N}$.

Proof. Consider a game $(N, v)$. For this proof, we introduce the following notations,

$$
\begin{aligned}
v^{-i}\left(S ; \pi\left((S+i)^{c}\right)\right) & =r_{i, S, \pi\left((S+i)^{c}\right)}^{N}\left(\left\{x_{i k}\right\}_{k=1}^{K}, x_{0}\right) \\
v^{-i}\left(S \cup N^{\prime} ; \pi^{t}\left(\left\{j_{1}, j_{2}, \ldots, j_{K}\right\}\right)\right) & =r_{i, S \cup N^{\prime}, \pi^{t}\left(\left\{j_{1}, \ldots, j_{K}\right\}\right)}^{N}\left(\left\{z_{i k}\right\}_{k=1}^{K}, z_{0}\right)
\end{aligned}
$$

where for each $k=1, \ldots K$.

$$
\begin{aligned}
\text { (i) } x_{i k} & =v\left(S ;\left\{S_{1}, \ldots, S_{k-1}, S_{k}+i, S_{k+1}, \ldots, S_{K}\right\}\right) \\
\text { (ii) } z_{i k} & =v\left(S \cup N^{\prime} ;\left\{\left\{j_{1}\right\}, \ldots,\left\{j_{k-1}\right\},\left\{j_{k}, i\right\},\left\{j_{k+1}\right\}, \ldots,\left\{j_{K}\right\}\right\}\right) \\
\text { (iii) } x_{0} & =v\left(S ;\left\{S_{1}, S_{2} \ldots, S_{K},\{i\}\right\}\right) \\
\text { (iv) } z_{0} & =v\left(S \cup N^{\prime} ; \pi^{t}\left(\left\{j_{1}, \ldots, j_{K}, i\right\}\right)\right)
\end{aligned}
$$

In this lemma, we want to show that two functions $r_{i, S, \pi\left((S+i)^{c}\right)}^{N}$ and $r_{i, S \cup N^{\prime}, \pi^{t}\left(\left(\left\{j_{1}, \ldots, j_{K}\right\}\right)\right)}^{N}$ are identical. Take any vector of arguments $\left(\left\{z_{i k}\right\}_{k=1}^{K}, z_{0}\right)$ of $\left.r_{i, S \cup N^{\prime}, \pi^{t}}^{N}\left(\left\{j_{1}, \ldots, j_{K}\right\}\right)\right)$. We need to show that $r_{i, S \cup N^{\prime}, \pi^{t}\left(\left(\left\{j_{1}, \ldots, j_{K}\right\}\right)\right)}^{N}\left(\left\{z_{i k}\right\}_{k=1}^{K}, z_{0}\right)=r_{i, S, \pi\left((S+i)^{c}\right)}^{N}\left(\left\{z_{i k}\right\}_{k=1}^{K}, z_{0}\right)$.

Let $N^{\prime}$ be dummy players of type 1 in $(N, v)$. First, note that the choice of $N^{\prime}$ to be dummy players of type 1 in $(N, v)$ does not impose any restrictions on the vector $\left(\left\{z_{i k}\right\}_{k=1}^{K}, z_{0}\right)$ and these numbers can be chosen arbitrarily. Hence, without loss of generality, we are allowed to set all members of $N^{\prime}$ as type 1 dummy players in $(N, v)$. However, we show that this choice induces the following equalities, $x_{i k}=z_{i k}$ for each $k=1, \ldots, K$ and $x_{0}=z_{0}$.

For all $k=1, \ldots K$,

$$
\begin{aligned}
z_{i k} & =v\left(S \cup N^{\prime} ;\left\{\left\{j_{1}\right\}, \ldots,\left\{j_{k-1}\right\},\left\{j_{k}, i\right\},\left\{j_{k+1}\right\}, \ldots,\left\{j_{K}\right\}\right\}\right) . \\
& =v\left(S ;\left\{S_{1}, \ldots, S_{k-1}, S_{k}+i, S_{k+1}, \ldots, S_{K}\right\}\right) \\
& =x_{i k}
\end{aligned}
$$


and $z_{0}=v\left(S \cup N^{\prime} ; \pi^{t}\left(\left\{j_{1}, \ldots, j_{K}, i\right\}\right)\right)=v\left(S ;\left\{S_{1}, S_{2} \ldots, S_{K},\{i\}\right\}\right)=x_{0}$.

Moreover, by the Weak Dummy Axiom, the members of $N^{\prime}$ remain dummy players of type 1 in $v^{-i}$. Hence, $v^{-i}\left(S ; \pi\left((S+i)^{c}\right)\right)=v^{-i}\left(S \cup N^{\prime} ; \pi^{t}\left(\left\{j_{1}, \ldots, j_{K}\right\}\right)\right)$.

Therefore for any $\left(\left\{z_{i k}\right\}_{k=1}^{K}, z_{0}\right)$,

$r_{i, S \cup N^{\prime}, \pi^{t}\left(\left\{j_{1}, \ldots, j_{K}\right\}\right)}^{N}\left(\left\{z_{i k}\right\}_{k=1}^{K}, z_{0}\right)=r_{i, S \cup N^{\prime}, \pi^{t}\left(\left\{j_{1}, \ldots, j_{K}\right\}\right)}^{N}\left(\left\{x_{i k}\right\}_{k=1}^{K}, x_{0}\right)=r_{i, S, \pi\left((S+i)^{c}\right)}^{N}\left(\left\{x_{i k}\right\}_{k=1}^{K}, x_{0}\right)$,

Hence, $r_{i, S, \pi\left((S+i)^{c}\right)}^{N}=r_{i, S \cup N^{\prime}, \pi^{t}\left(\left\{j_{1}, \ldots, j_{K}\right\}\right)}^{N}$.

We now show that Scale Invariance and Sign Independence imply a stronger form of Scale Invariance.

Definition 16 A restriction operator satisfies Scale Invariance* if if for all $(N, v),\left(N, v^{\prime}\right) \in$ $\mathbb{V}$, if $v=a+b v^{\prime}$ for some $a, b$ then for all $i \in N, v^{-i}=a+b v^{-i}$.

That is, unlike Scale Invariance, the antecedent of Scale Invariance* does not impose the requirement that $b>0$.

Lemma 2 Let $r$ be a restriction operator satisfying Scale Invariance and Sign Independence. Then $r$ satisfies Scale Invariance*.

Proof. Consider games $(N, v),(N, \bar{v})$, where $v, \bar{v}$ are such that $v=-b \bar{v}$, where $b>0$. Because $r$ satisfies Scale Invariance, it suffices to show $v^{-i}=-b \bar{v}^{-i}$. For any $S \subseteq N$, let

$$
a_{S}=\min _{\pi\left(S^{c}\right) \in \Pi_{S^{c}}} v\left(S ; \pi\left(S^{c}\right)\right)
$$

and

$$
a=\min _{S \subseteq N} a_{S}
$$

Then, $-a+v \geq 0$. By Scale Invariance, for all $i \in N,(-a+v)^{-i}=-a+v^{-i}$.

Now from Sign Independence, $a-v \leq 0$, and Scale Invariance,

$$
(a-v)^{-i}=-(-a+v)^{-i}=-\left(-a+v^{-i}\right)=a-v^{-i} .
$$

Also $a-v=a+b \bar{v}$ and by Scale Invariance, $(a+b \bar{v})^{-i}=a+b \bar{v}^{-i}$.

Now we must have $-v^{-i}=b \bar{v}^{-i}$. So, Scale Invariance* is satisfied.

The next lemma shows that the removal of a type 1 dummy player from a game does not affect the other players.

Lemma 3 Let $r$ be a restriction operator satisfying Scale Invariance*. Let $(N, v)$ be a game where $i$ is a type 1 dummy player. Choose any $S \subseteq N-i$ and a partition $\pi(N-(S+i))$. Then $v^{-i}(S ; \pi(N-(S+i)))=v(S+i ; \pi(N-(S+i)))$. 
Proof. Since $i$ is a type 1 dummy player, we have $v(S ; \pi)=v(S+i ; \pi(N-(S+i)))$ for all $\pi \in \pi^{+i}(N-(S+i))$. Thus

$$
\begin{aligned}
v^{-i}(S ; \pi(N-(S+i))) & =r_{i, S, \pi(N-(S+i))}^{N}\left((v(S ; \pi))_{\pi \in \pi^{+i}(N-(S+i))}\right) \\
& =r_{i, S, \pi(N-(S+i))}^{N}\left(\left(v(S+i ; \pi(N-(S+i)))_{\pi \in \pi^{+i}(N-(S+i))}\right)\right. \\
& =v(S+i ; \pi(N-(S+i)))
\end{aligned}
$$

where the last equality is a consequence of Scale Invariance*: note that for the null game $(\hat{v}, N)$ (where $\hat{v}\left(S ; \pi\left(S^{c}\right)\right)=0$ for all embedded coalitions $\left(S ; \pi\left(S^{c}\right)\right.$ ) we have $\hat{v}=-\hat{v}$ and from Scale Invariance* we obtain $\hat{v}^{-i}=-\hat{v}^{-i}$ and $\hat{v}^{-i}$ must be the null

game on $N \backslash\{i\}$; hence, by Scale Invariance*, $r_{i, S, \pi(N-(S+i))}^{N}\left(\left(v(S+i ; \pi(N-(S+i)))_{\pi \in \pi^{+i}(N-(S+i))}\right)=\right.$ $v\left(S+i ; \pi(N-(S+i))+r_{i, S, \pi(N-(S+i))}^{N}\left((0)_{\pi \in \pi^{+i}(N-(S+i))}\right)=v(S+i ; \pi(N-(S+i))\right.$, the desired conclusion.

We use Lemma 3 to prove that Limited Independence is implied by Path Independence, Scale Invariance* and the Weak Dummy Axiom. We will use limited Independence in the proof of Theorem 2.

Lemma 4 Let $r$ be a restriction operator satisfying Path Independence, the Weak Dummy Axiom and Scale Invariance*. Then $r$ satisfies Limited Independence.

Proof. Let $R=N_{2}-N_{1}$. Let us choose a game $\left(N_{2}, v\right)$ in which $R$ is the set of type 1 dummy players. Note that $v^{-R}$ is a game on $N_{1}$. Take any $S \subseteq\left(N_{1}-i\right)$ and any partition $\pi(T)$, where $T=N_{1}-(S+i)$. Now consider the subgame of $v$ on $\left(N_{2}-i\right)$, generated by $r$. By repeated use of Lemma 3 and the Weak Dummy Axiom, we get

$v^{-i}(S+R ; \pi(T))=r_{i, S+R, \pi(T)}^{N_{2}}\left((v(S+R ; \pi))_{\pi \in \pi^{+i}(T)}\right)=r_{i, S+R, \pi(T)}^{N_{2}}\left(\left(v^{-R}(S ; \pi)\right)_{\pi \in \pi^{+i}(T)}\right)$

Moreover, the Weak Dummy Axiom ensures that $R$ is still the set of type 1 dummy player in $v^{-i}$. Once again by repeated use of Lemma 3 and the Weak Dummy Axiom, we get, $v^{-i}(S+R ; \pi(T))=v^{-(i+R)}(S ; \pi(T))$. Since $r$ is Path Independent, $v^{-(i+R)}$ is also the subgame of $v^{-R}$ on $\left(N_{1}-i\right)$. That is, $v^{-(i+R)}(S ; \pi(T))=$ $r_{i, S, \pi(T)}^{N_{1}}\left(\left(v^{-R}(S ; \pi)\right)_{\pi \in \pi^{+i}(T)}\right)$. Hence, from (4),

$$
r_{i, S+R, \pi(T)}^{N_{2}}\left(\left(v^{-R}(S ; \pi)\right)_{\pi \in \pi^{+i}(T)}\right)=r_{i, S, \pi(T)}^{N_{1}}\left(\left(v^{-R}(S ; \pi)\right)_{\pi \in \pi^{+i}(T)}\right)
$$

Note that our choice of dummy players does not impose any restriction on the vector $\{v(S+R ; \pi)\}_{\pi \in \pi^{+i}(T)}$ or equivalently on $\left\{v^{-R}(S ; \pi)\right\}_{\pi \in \pi^{+i}(T)}$. Therefore $r_{i, S, \pi(T)}^{N_{1}}=r_{i, S+R, \pi(T)}^{N_{2}}$.

Below we will use sometimes $r_{i}$ instead of $r_{i, S, \pi\left(S^{c}\right)}^{N}$.

\section{Proof of Theorem 2:}


Let $r$ be a restriction operator satisfying Path Independence, the Weak Dummy Axiom, Scale Invariance and Sign Independence. By Lemma 2 and Lemma 4, $r$ satisfies Scale Invariance* and Limited Independence.

Choose any $i \in N$ and $S \subseteq N-i$. We prove the theorem by induction on the number of elements in $\pi^{+i}\left(S^{c}\right)$.

Suppose $\left|\pi^{+i}\left(S^{c}\right)\right|=1$. Then $v^{-i}(S)=r_{i}(v(S ;\{i\}))$. By Scale Invariance*, $r_{i}(v(S ; i))=v(S ; i) .{ }^{22}$ Hence the induction hypothesis is satisfied.

Now, suppose $\left|\pi^{+i}\left(S^{c}\right)\right|=2$. Let us first show that the induction hypothesis holds for embedded coalitions of the type $(S ; j)$. From our specification,

$$
v^{-i}(N-i j ; j)=r_{i}(v(N-i j ; j i), v(N-i j ; j, i))
$$

Let $r_{i}(1,0)=\theta_{i}^{N}$. By Scale Invariance*,

$$
\begin{aligned}
r_{i}(v(N-i j ; j i), v(N-i j ; j, i)) & =v(N-i j ; j, i)+(v(N-i j ; j i)-v(N-i j ; j, i)) r_{i}(1,0) \\
& =\theta_{i}^{N} v(N-i j ; j i)+\left(1-\theta_{i}^{N}\right) v(N-i j ; j, i)
\end{aligned}
$$

We have already proved that $v^{-i j}(S)=v^{-i}(S ; j)$. Hence,

$$
v^{-i j}(N-i j)=\theta_{i}^{N} v(N-i j ; j i)+\left(1-\theta_{i}^{N}\right) v(N-i j ; j, i)
$$

By similar arguments,

$$
v^{-j i}(N-i j)=\theta_{j}^{N} v(N-i j ; j i)+\left(1-\theta_{j}^{N}\right) v(N-i j ; j, i)
$$

However, Path Independence implies $v^{-i j}(N-i j)=v^{-j i}(N-i j)$. Therefore $\theta_{i}^{N}=$ $\theta_{j}^{N}=\theta^{N}$ (say). Using the same argument on $\mathbb{N}$, we get,

$$
r_{i}^{\mathbb{N}}(v(\mathbb{N}-i j ; j i), v(\mathbb{N}-i j ; j, i))=\theta^{\mathbb{N}} v(\mathbb{N}-i j ; j i)+\left(1-\theta^{\mathbb{N}}\right) v(\mathbb{N}-i j ; j, i)
$$

By Lemma 4, $r_{i, \mathbb{N}-i j, \pi^{t}(\{j\})}^{\mathbb{N}}=r_{i, N-i j, \pi^{t}(\{j\})}^{N}$, hence $\theta^{N}=\theta^{\mathbb{N}}$. Therefore the coefficient does not depend upon $N$ and we will simply represent it by $\theta$.

We can use Lemma 1 to extend our analysis for all partitions with $\left|\pi^{+i}\left(S^{c}\right)\right|=2$.

So, the theorem is true when $\pi^{+i}\left(S^{c}\right)$ has no more than two elements. Suppose the theorem is true for all partitions when $\pi^{+i}\left(S^{c}\right)$ has $K$ or less elements for some $K$. We want to show that the theorem remains true when $\pi^{+i}\left(S^{c}\right)$ has $K+1$ elements.

Choose $N$ such that $|N| \geq K+2$. Choose $i \in N$ and $S \subset N-i$ such that $\pi\left((S+i)^{c}\right)=\pi^{t}\left((S+i)^{c}\right)$ has exactly $K$ elements. Hence, $\pi^{+i}\left((S+i)^{c}\right)$ has $K+1$ elements.

Our first aim is to calculate $v^{-i}\left(S ; \pi^{t}\left((S+i)^{c}\right)\right)$, with the help of the induction hypothesis.

From our specification,

$$
v^{-i}\left(S ; \pi^{t}\left((S+i)^{c}\right)\right)=r_{i}\left(\left\{v\left(S ; i k, \pi^{t}\left((S+i k)^{c}\right)\right)\right\}_{k \notin(S+i)}, v\left(S ; \pi^{t}\left(S^{c}\right)\right)\right)
$$

\footnotetext{
${ }^{22}$ The same argument as at the end of the proof of Lemma 3 establishes this.
} 
Denoting $v\left(S ; i k, \pi^{t}\left((S+i k)^{c}\right)\right)=x_{i k}$ and $v\left(S ; \pi^{t}\left(S^{c}\right)\right)=x_{0}$, we can rewrite the previous equation as

$$
v^{-i}\left(S ; \pi^{t}\left((S+i)^{c}\right)\right)=r_{i}\left(\left\{x_{i k}\right\}_{k \notin S+i}, x_{0}\right)
$$

Similarly, for any $m \neq i$, we can write $v^{-m}\left(S ; \pi^{t}\left((S+m)^{c}\right)\right)$ as

$$
v^{-m}\left(S ; \pi^{t}\left((S+m)^{c}\right)\right)=r_{m}\left(\left\{x_{m k}\right\}_{k \notin S+m}, x_{0}\right)
$$

where $x_{m k}=v\left(S ; m k, \pi^{t}\left((S+m k)^{c}\right)\right)$, and $x_{i m}=x_{m i}$.

We can use the induction hypothesis to calculate $v^{-i m}\left(S ; \pi^{t}\left((S+i m)^{c}\right)\right)$. Indeed,

$$
\begin{aligned}
& v^{-i m}\left(S ; \pi^{t}\left((S+i m)^{c}\right)\right) \\
& =\theta \sum_{k \notin(S+i m)} v^{-i}\left(S ; m k, \pi^{t}\left((S+i m k)^{c}\right)\right)+(1-K \theta) v^{-i}\left(S ; \pi^{t}\left((S+i)^{c}\right)\right) \\
& =\theta \sum_{k \notin(S+i m)}\left[\theta \sum_{\ell \notin(S+i m k)} v\left(S ; m k, i \ell, \pi^{t}\left((S+i m k \ell)^{c}\right)\right)+\theta v\left(S ; m k i, \pi^{t}\left((S+i m k)^{c}\right)\right)\right. \\
& \left.\quad+(1-K \theta) v\left(S ; m k, \pi^{t}\left((S+m k)^{c}\right)\right)\right]+(1-K \theta) v^{-i}\left(S ; \pi^{t}\left((S+i)^{c}\right)\right)
\end{aligned}
$$

Similarly,

$$
\begin{aligned}
& v^{-m i}\left(S ; \pi^{t}\left((S+i m)^{c}\right)\right) \\
& =\theta \sum_{k \notin(S+i m)}\left[\theta \sum _ { \ell \notin ( S + i m k ) } v \left(S ; i k, m \ell, \pi^{t}\left((S+i m k \ell)^{c}\right)+\theta v\left(S ; m k i, \pi^{t}\left((S+i m k)^{c}\right)\right)\right.\right. \\
& \left.\quad+(1-K \theta) v\left(S ; i k, \pi^{t}\left((S+i k)^{c}\right)\right)\right] \\
& \quad+(1-K \theta) v^{-m}\left(S ; \pi^{t}\left((S+m)^{c}\right)\right)
\end{aligned}
$$

From Path Independence,

$$
v^{-i m}\left(S ; \pi^{t}\left((S+i m)^{c}\right)\right)=v^{-m i}\left(S ; \pi^{t}\left((S+i, m)^{c}\right)\right)
$$

Also,

$$
\begin{aligned}
& \sum_{k \notin(S+i m)} \sum_{\ell \notin(S+i m k)} v\left(S ; m k, i \ell, \pi^{t}\left((S+i m k \ell)^{c}\right)\right) \\
= & \sum_{\ell \notin(S+i m)} \sum_{k \notin(S+i m \ell)} v\left(S ; m k, i \ell, \pi^{t}\left((S+i m k \ell)^{c}\right)\right) \\
= & \sum_{k \notin(S+i m)} \sum_{\ell \notin(S+i m k)} v\left(S ; i k, m \ell, \pi^{t}\left((S+i m k \ell)^{c}\right)\right)
\end{aligned}
$$

Thus,

$$
\begin{aligned}
& {\left[v^{-i}\left(S ; \pi^{t}\left((S+i)^{c}\right)\right)-v^{-m}\left(S ; \pi^{t}\left((S+m)^{c}\right)\right)\right] } \\
= & \theta \sum_{k \notin(S+i m)}\left[v\left(S ; i k, \pi^{t}\left((S+i k)^{c}\right)\right)-v\left(S ; m k, \pi^{t}\left((S+m k)^{c}\right)\right)\right] \\
= & \theta \sum_{k \notin(S+i m)}\left(x_{i k}-x_{m k}\right)
\end{aligned}
$$


By choosing $x_{i k}=x_{m k} \equiv x_{k}$ for all $k \notin(S+i m)$, we obtain

$$
r_{i}\left(\left\{x_{i k}\right\}_{k \notin(S+i)}, x_{0}\right)=r_{m}\left(\left\{x_{m k}\right\}_{k \notin(S+m)}, x_{0}\right) \equiv r\left(\left\{x_{k}\right\}_{k \notin(S+i)}, x_{0}\right)
$$

So, from (8),

$$
r\left(\left\{x_{i k}\right\}_{k \notin(S+i)}, x_{0}\right)=\theta \sum_{k \notin(S+i m)}\left(x_{i k}-x_{m k}\right)+r\left(\left\{x_{m k}\right\}_{k \notin(S+m)}, x_{0}\right)
$$

Let $p \notin(S+i m)$ be a dummy player of type 1 in $v$. Then, $p$ is also a dummy player in $v^{-i}$, and so

$$
v^{-i}\left(S ; \pi^{t}\left((S+i)^{c}\right)\right)=v^{-i}\left(S+p ; \pi^{t}\left((S+i p)^{c}\right)\right)
$$

From the induction hypothesis,

$v^{-i}\left(S+p ; \pi^{t}\left((S+i p)^{c}\right)\right)=\theta \sum_{k \notin(S+i p)} v\left(S+p ; k i, \pi^{t}\left((S+i p k)^{c}\right)\right)+(1-K \theta) v\left(S+p ; \pi^{t}\left((S+p)^{c}\right)\right)$

Since $p$ is a dummy player, $v\left(S+p ; k i, \pi^{t}\left((S+i k p)^{c}\right)\right)=v\left(S ; k i, \pi^{t}\left((S+i k)^{c}\right)\right)$ and $v\left(S+p ; \pi^{t}\left((S+p)^{c}\right)\right)=v\left(S ; \pi^{t}\left(S^{c}\right)\right)$. Also, $v\left(S ; p i, \pi^{t}\left((S+p)^{c}\right)\right)=v\left(S ; \pi^{t}\left(S^{c}\right)\right)$. So,

$$
\begin{aligned}
v^{-i}\left(S ; \pi^{t}\left((S+i)^{c}\right)\right) & =v^{-i}\left(S+p ; \pi^{t}\left((S+i, p)^{c}\right)\right) \\
& =\theta \sum_{k \notin(S+i p)} v\left(S ; k i, \pi^{t}\left((S+i k)^{c}\right)\right)+(1-K \theta) v\left(S ; \pi^{t}\left(S^{c}\right)\right) \\
& =\theta \sum_{k \notin(S+i)} v\left(S ; k i, \pi^{t}\left((S+i k)^{c}\right)\right)+(1-(K+1) \theta) v\left(S ; \pi^{t}(S)\right)
\end{aligned}
$$

That is,

$$
r\left(\left\{x_{i k}\right\}_{k \notin(S+i)}, x_{0}\right)=\theta \sum_{k \notin(S+i)} x_{i k}+(1-(K+1) \theta) x_{0}
$$

Notice that this equality is proved under the assumption that $x_{i p}=x_{0}$, and so we do not as yet have a general expression for $r$.

Finally, from (10),

$$
\begin{aligned}
r\left(\left\{x_{m k}\right\}_{k \notin(S+m)}, x_{0}\right) & =\theta \sum_{k \notin(S+i)} x_{i k}+(1-(K+1) \theta) x_{0}-\theta \sum_{k \notin(S+i m)}\left(x_{i k}-x_{m k}\right) \\
& =x_{m i}+\theta \sum_{k \notin(S+i m)} x_{m k}+(1-(K+1) \theta) x_{0} \\
& =\theta \sum_{k \notin(S+m)} x_{m k}+(1-(K+1) \theta) x_{0}
\end{aligned}
$$

Hence,

$$
v^{-i}\left(S ; \pi^{t}\left((S+i)^{c}\right)\right)=\theta \sum_{k \notin(S+i)} v\left(S ; i k, \pi^{t}\left((S+i, k)^{c}\right)\right)+(1-(K+1) \theta) v\left(S ; \pi^{t}(S)\right)
$$


We can use Lemma 1 to extend our analysis for all partitions with $\left|\pi^{+i}(S)\right|=$ $K+1$. This completes the induction step.

In order to complete the proof of the theorem, we have to demonstrate the implication of Non-negativity. Consider a game $(N, v) \in \mathbb{V}$ such that for all $S \subseteq N$,

(i) $v(S ; \pi)=0$ if there is some $i \in S^{c}$ such that $\{i\} \in \pi$.

(ii) $v(S ; \pi)>0$ otherwise.

Now, suppose $\theta<0$. Choose any $S \subset N$ and any $i \notin S$. Then,

$$
v^{-i}\left(S ; \pi\left((S \cup\{i\})^{c}\right)\right)=\theta \sum_{\pi \in \pi^{+i}\left((S \cup\{i\})^{c}\right)} v(S ; \pi)<0
$$

where the inequality follows from the fact that $v(S ; \pi)=0$ if $\{i\} \in \pi$. But, this violates Non-negativity, and so $\theta \geq 0$.

Now, consider a $(\mathbb{N}, v) \in \mathbb{V}$ such that $v\left(i ; \pi^{t}(\mathbb{N} \backslash\{i\})\right)>0$ and $v(S ; \pi(\mathbb{N} \backslash S))=0$ otherwise. Then $v^{-j}\left(i ; \pi^{t}(\mathbb{N} \backslash\{i j\})\right)=(1-(|\mathbb{N}|-2) \theta) v\left(i ; \pi^{t}(\mathbb{N} \backslash\{i\})\right)$ and the upper bound follows from Non-negativity. This concludes the proof of Theorem 2 .

\section{Proof of Theorem 3:}

Suppose the restriction operator satisfies Path Independence, the Strong Dummy Axiom, Scale Invariance and Monotonicity. We show that for all games $(N, v)$ and all $i \in N$,

$$
v^{-i}\left(S ; \pi^{t}\left((S+i)^{c}\right)\right)=v\left(S ; \pi^{t}\left((S+i)^{c}\right) \cup\{\{i\}\}\right)
$$

That is, the Strong Dummy Axiom implies that $\theta=0$. This can be checked as follows. Let $N=\{1,2,3,4\}$. By Theorem 2,

$$
\begin{aligned}
v^{-4}(\{1,2\} ;\{3\}) & =\theta v(\{1,2\} ;\{3,4\})+(1-\theta) v(\{1,2\} ;\{3\},\{4\}) \\
v^{-4}(\{1\} ;\{2\},\{3\}) & =\theta v(\{1\} ;\{2\},\{3,4\})+\theta v(\{1\} ;\{2,4\},\{3\})+(1-2 \theta) v(\{1\} ;\{2\},\{3\},\{4\})
\end{aligned}
$$

Suppose 2 is a dummy player of type 2 in $v$. By the Strong Dummy Axiom, player 2 must be a type 2 dummy player in $v^{-4}$. Thus, $v^{-4}(\{1,2\} ;\{3\})=v^{-4}(\{1\} ;\{2\},\{3\})$. This is only possible if $\theta=0$, because the assumption of type 2 dummy player does not impose any restriction on $v(\{1\} ;\{2,4\},\{3\})$. Hence the result. It is easy to check that the sing operator will satisfy all the axioms.

Before proving Theorem 5, we introduce a property which is of instrumental importance for this proof. As we show, this property is satisfied by all of the examples given in the main text.

Definition 17 A restriction operator $r$ is Regular if for all solutions $\varphi$, for all $(N, v) \in \mathbb{V}$, and $S \subset N$, the reduced game $v_{S}^{\varphi, r}$ satisfies the following for all $R \subseteq S$

$$
\left(v_{S}^{\varphi, r}\right)^{-S \backslash R, r}(R)=v^{-S \backslash R, r}\left(R \cup S^{c}\right)-\sum_{k \in S^{c}} \varphi_{k}\left(R \cup S^{c}, v^{-S \backslash R, r}\right)
$$


Lemma 5 Let $r$ be a restriction operator satisfying Path Independence, Limited Independence and Translation Invariance. Then $r$ satisfies Regularity.

Proof. Let $\varphi$ be a solution, $(N, v) \in \mathbb{V}$, and $S \subset N$. Then the reduced game $\left(S, v_{S}^{\varphi, r}\right) \in \mathbb{V}$. Choose any $R \subseteq S$. We will prove the result by induction on $|S \backslash R|$. In fact, to use induction, we will prove a slightly general result than required for Regularity. This is as follows. Choose any $R_{1} \subseteq R$ and $\pi\left(R \backslash R_{1}\right)$. We will show that

$\left(v_{S}^{\varphi, r}\right)^{-S \backslash R, r}\left(R_{1} ; \pi\left(R \backslash R_{1}\right)\right)=v^{-S \backslash R, r}\left(R_{1} \cup S^{c} ; \pi\left(R \backslash R_{1}\right)\right)-\sum_{k \in S^{c}} \varphi_{k}\left(R_{1} \cup S^{c}, v^{-\left(S \backslash R_{1}\right), r}\right)$

where $S^{c}=N \backslash S$. Note that Regularity is a special case of the above statement, where $R=R_{1}$.

First, let us prove our claim when $|S \backslash R|=1$. Suppose $S \backslash R=\{i\}$. Then,

$$
\begin{aligned}
& \left(v_{S}^{\varphi, r}\right)^{-i, r}\left(R_{1} ; \pi\left(R \backslash R_{1}\right)\right) \\
& =r_{i, R_{1}, \pi\left(R \backslash R_{1}\right)}^{S}\left(\left(v_{S}^{\varphi, r}\left(R_{1} ; \pi\right)\right)_{\pi \in \pi^{+i}\left(R \backslash R_{1}\right)}\right) \\
& =r_{i, R_{1}, \pi\left(R \backslash R_{1}\right)}^{S}\left(\left(v\left(R_{1} \cup S^{c} ; \pi\right)-\sum_{k \in S^{c}} \varphi_{k}\left(R_{1} \cup S^{c}, v^{-\left(S \backslash R_{1}\right), r}\right)\right)_{\pi \in \pi^{+i}\left(R \backslash R_{1}\right)}\right) \\
& =r_{i, R_{1}, \pi\left(R \backslash R_{1}\right)}^{S}\left(\left(v\left(R_{1} \cup S^{c} ; \pi\right)\right)_{\pi \in \pi^{+i}\left(R \backslash R_{1}\right)}\right)-\sum_{k \in S^{c}} \varphi_{k}\left(R_{1} \cup S^{c}, v^{-\left(S \backslash R_{1}\right), r}\right) \\
& =r_{i, R_{1} \cup S^{c}, \pi\left(R \backslash R_{1}\right)}^{N}\left(\left(v\left(R_{1} \cup S^{c} ; \pi\right)\right)_{\pi \in \pi^{+i}\left(R \backslash R_{1}\right)}\right)-\sum_{k \in S^{c}} \varphi_{k}\left(R_{1} \cup S^{c}, v^{-\left(S \backslash R_{1}\right), r}\right) \\
& =v^{-i, r}\left(R_{1} \cup S^{c} ; \pi\left(R \backslash R_{1}\right)\right)-\sum_{k \in S^{c}} \varphi_{k}\left(R_{1} \cup S^{c}, v^{-\left(S \backslash R_{1}\right), r}\right)
\end{aligned}
$$

where the first and the fifth equality use the definition of the restriction operator, the second uses the definition of the reduced game $v_{S}^{\varphi, r}$, the third and the fourth follow from Translation Invariance and Limited Independence of $r$, respectively.

Now, suppose that our claim is true for $|S \backslash R|<m$. We will show that the same is true when $|S \backslash R|=m$. Suppose $i \in S \backslash R$. By Path independence, $\left(v_{S}^{\varphi, r}\right)^{-S \backslash R, r}$ can be obtained by removing players of $S \backslash R$ in any sequence. Thus without loss of generality we can remove $i$ after everyone else. Hence, $\left(v_{S}^{\varphi, r}\right)^{-S \backslash R, r}=$ $\left(\left(v_{S}^{\varphi, r}\right)^{-(S \backslash(R \cup\{i\})), r}\right)^{-i, r}$. For notational convenience, we will denote $\left(v_{S}^{\varphi, r}\right)^{-(S \backslash(R \cup\{i\})), r}$ as $\bar{v}$. Note that $\bar{v}$ is a game on $R \cup\{i\}$ and $S \backslash(R \cup\{i\})<m$. Therefore the induction 
hypothesis is applicable on $\bar{v}$. Then

$$
\begin{aligned}
& \left(v_{S}^{\varphi, r}\right)^{-S \backslash R, r}\left(R_{1} ; \pi\left(R \backslash R_{1}\right)\right) \\
& =(\bar{v})^{-i, r}\left(R_{1} ; \pi\left(R \backslash R_{1}\right)\right) \\
& =r_{i, R_{1}, \pi\left(R \backslash R_{1}\right)}^{R \cup\left\{\left(\bar{v}\left(R_{1} ; \pi\right)\right)_{\pi \in \pi^{+i}\left(R \backslash R_{1}\right)}\right)} \\
& =r_{i, R_{1}, \pi\left(R \backslash R_{1}\right)}^{R \cup\{i\}}\left(\left(v^{-(S \backslash(R \cup\{i\})), r}\left(R_{1} \cup S^{c} ; \pi\right)-\sum_{k \in S^{c}} \varphi_{k}\left(R_{1} \cup S^{c}, v^{-\left(S \backslash R_{1}\right), r}\right)\right)_{\pi \in \pi^{+i}\left(R \backslash R_{1}\right)}\right) \\
& =r_{i, R_{1}, \pi\left(R \backslash R_{1}\right)}^{R \cup i\}}\left(\left(v^{-(S \backslash(R \cup\{i\})), r}\left(R_{1} \cup S^{c} ; \pi\right)\right)_{\pi \in \pi^{+i}\left(R \backslash R_{1}\right)}\right)-\sum_{k \in S^{c}} \varphi_{k}\left(R_{1} \cup S^{c}, v^{-\left(S \backslash R_{1}\right), r}\right) \\
& =r_{i, R_{1} \cup S^{c}, \pi\left(R \backslash R_{1}\right)}^{R \cup\{i\} \cup S^{c}}\left(\left(v^{-(S \backslash(R \cup\{i\})), r}\left(R_{1} \cup S^{c} ; \pi\right)\right)_{\pi \in \pi^{+i}\left(R \backslash R_{1}\right)}\right)-\sum_{k \in S^{c}} \varphi_{k}\left(R_{1} \cup S^{c}, v^{-\left(S \backslash R_{1}\right), r}\right) \\
& =\left(v^{-(S \backslash(R \cup\{i\})), r}\right)^{-i, r}\left(R_{1} \cup S^{c} ; \pi\left(R \backslash R_{1}\right)\right)-\sum_{k \in S^{c}} \varphi_{k}\left(R_{1} \cup S^{c}, v^{-\left(S \backslash R_{1}\right), r}\right) \\
& =v^{-S \backslash R, r}\left(R_{1} \cup S^{c} ; \pi\left(R \backslash R_{1}\right)\right)-\sum_{k \in S^{c}} \varphi_{k}\left(R_{1} \cup S^{c}, v^{-\left(S \backslash R_{1}\right), r}\right)
\end{aligned}
$$

where the third equality follows from the induction hypothesis. The rest is exactly the same steps as those in $|S \backslash R|=1$. Hence, $r$ satisfies Regularity.

\section{Proof of Theorem 5:}

Throughout the proof, fix some restriction operator $r$ satisfying Path Independence, Limited Independence and Translation Invariance. By Lemma 5, $r$ satisfies Regularity. Since $r$ is fixed, we omit all reference to $r$ in the rest of the proof.

We first show that $S h$ satisfies consistency. Choose any $(N, v) \in \mathbb{V}$, and $S \subset N$. We need to show that for all $i \in S$,

$$
S h_{i}(N, v)=S h_{i}\left(S, v_{S}^{S h}\right) \text { where } v_{S}^{S h} \text { is the reduced game on } S .
$$

For all $R \subseteq S$, and $\pi \in \Pi_{S \backslash R}$,

$$
v_{S}^{S h}(R ; \pi)=v\left(R \cup S^{c} ; \pi\right)-\sum_{k \in S^{c}} S h_{k}\left(R \cup S^{c}, v^{-S \backslash R}\right)
$$

where $v^{-S \backslash R}$ is the subgame (of $v$ ) on the player set $S^{c} \cup R$.

Let $w, w_{S}$ and $w^{-S \backslash R}$ be the characteristic function games associated with $v, v_{S}^{S h}$ and $v^{-S \backslash R}$ respectively. Let $\left(v_{S}^{S h}\right)^{-(S \backslash R)}$ denote the subgame of $v_{S}^{S h}$ on the player set $R$. Since the restriction operator satisfies Regularity,

$$
\left(v_{S}^{S h}\right)^{-(S \backslash R)}(R)=v^{-(S \backslash R)}\left(R \cup S^{c}\right)-\sum_{k \in S^{c}} S h_{k}\left(R \cup S^{c}, v^{-(S \backslash R)}\right)
$$


Hence,

$$
\begin{aligned}
w_{S}(R) & =\left(v_{S}^{S h}\right)^{-(S \backslash R)}(R) \\
& =v^{-(S \backslash R)}\left(R \cup S^{c}\right)-\sum_{k \in S^{c}} S h_{k}\left(R \cup S^{c}, v^{-(S \backslash R)}\right) \\
& =w\left(R \cup S^{c}\right)-\sum_{k \in S^{c}} S h_{k}\left(R \cup S^{c}, w^{-(S \backslash R)}\right) \\
& =w\left(R \cup S^{c}\right)-\sum_{k \in S^{c}} S h_{k}\left(R \cup S^{c},\left.w\right|_{R \cup S^{c}}\right) \\
& =w_{S}^{S h}(R) .
\end{aligned}
$$

Therefore for all $i \in S$,

$$
S h_{i}\left(S, v_{S}^{S h}\right)=S h_{i}\left(S, w_{S}\right)=S h_{i}\left(S, w_{S}^{S h}\right)=S h_{i}(N, w)=S h_{i}(N, v) \text {, }
$$

where the third equality follows from consistency of the Shapley value on TU games. That is, $S h$ satisfies consistency.

It is obvious that $S h$ satisfies the standard game property. We now prove the converse by showing that there can be only one solution satisfying the standard property on two-person games and $r$-consistency for any given $r$.

First, if $\varphi$ is a solution satisfying these two properties, then $\varphi$ must be efficient. The proof of this is very similar to that in Hart and Mas-Colell [5].

So, if $n=1$, then $\varphi_{i}(i, v)=v(i)$ and so there must be a unique solution. Similarly, the standard property on two-person games ensures that there is a unique solution on all two-person games. Suppose now that there is a unique solution satisfying consistency and the standard property on two-person games on all games $(N, v) \in \mathbb{V}$ when $|N|<m$.

Suppose now that $|N|=m$ and both $\varphi$ and $\psi$ are two different solutions satisfying these two properties. Since $\varphi$ and $\psi$ are different solutions, and both are efficient, there must exist $(N, v) \in \mathbb{V}$ and $i, j \in N$ such that $\varphi_{i}(N, v)>\psi_{i}(N, v)$, while $\varphi_{j}(N, v)<\psi_{j}(N, v)$. Define $S=\{i, j\}$. Now,

$$
\begin{aligned}
& v_{S}^{\varphi}(i)=v(N-j ; j)-\sum_{k \neq i, j} \varphi_{k}\left(N-j, v^{-j}\right) \\
& v_{S}^{\psi}(i)=v(N-j ; j)-\sum_{k \neq i, j} \psi_{k}\left(N-j, v^{-j}\right)
\end{aligned}
$$

From the induction hypothesis, $\varphi$ and $\psi$ coincide on $\left(N-j, v^{-j}\right)$. Hence,

$$
\sum_{k \neq i, j} \psi_{k}\left(N-j, v^{-j}\right)=\sum_{k \neq i, j} \varphi_{k}\left(N-j, v^{-j}\right)
$$

This implies that

$$
v_{S}^{\varphi}(i)=v_{S}^{\psi}(i)
$$


Similarly,

$$
v_{S}^{\varphi}(j)=v_{S}^{\psi}(j)
$$

Then, from consistency,

$$
\varphi_{i}\left(S, v_{S}^{\varphi}\right)=\varphi_{i}(N, v)>\psi_{i}(N, v)=\psi_{i}\left(S, v_{S}^{\psi}\right)
$$

So, by the standard property on two-person games, $\varphi_{j}\left(S, v_{S}^{\varphi}\right) \geq \psi_{j}\left(S, v_{S}^{\psi}\right)$. This contradicts the assumption that $\varphi_{j}(N, v)<\psi_{j}(N, v)$.

\section{Proof of Corollary 1:}

We show that the max operator satisfies Path Independence and Translation Invariance. Again, we omit any explicit reference to the operator in order to simplify the notation.

Fix any game $(N, v)$.

To check that the max operator satisfies Path Independence, choose any $i, j \in N$, and $S \subset N-i j$. Let $\pi \equiv\left\{T_{1}, \ldots, T_{K}\right\}$ be any partition of $(S+i j)^{c}$. Let $\Pi^{\prime}$ denote the set of partitions of $S^{c}$ satisfying

$$
\Pi^{\prime}=\left\{\pi^{\prime} \in \Pi_{S^{c}} \mid T^{\prime} \in \pi^{\prime} \rightarrow T_{k} \subset T^{\prime} \cup\{i, j\} \text { for some } k=1, \ldots K\right\}
$$

That is, $\Pi^{\prime}$ is the set of partitions of $S^{c}$ where each of $i$ and $j$ can join any of the elements of $\pi$, or remain single or form the set $\{i, j\}$. Then,

$$
v^{-i j}(S ; \pi)=\max _{\pi^{\prime} \in \Pi^{\prime}}\left[v\left(S ; \pi^{\prime}\right)\right]=v^{-j i}(S ; \pi)
$$

This shows that the max operator satisfies Path Independence.

To check that max satisfies Translation Invariance, let $x_{1}, \ldots, x_{k}$ and $c$ be any real numbers. Then,

$$
\max _{l=1, \ldots, k}\left[x_{l}-c\right]=\max _{l=1, \ldots, k}\left[x_{l}\right]-c .
$$

This shows that max satisfies Translation Invariance.

It is easy to see that the max operator satisfies Limited Independence.

\section{Proof of Corollary 2:}

We show that the sing operator satisfies Path Independence. It is straightforward to verify that it satisfies Limited Independence and Translation Invariance. Again, we omit any explicit reference to the operator in order to simplify the notation.

Fix any game $(N, v)$. Choose any $i, j \in N$, and $S \subset N-i j$. Let $\pi$ be any partition of $(S+i j)^{c}$. Then,

$$
v^{-i j}(S ; \pi)=v(S ; \pi \cup\{\{i\},\{j\}\})=v^{-j i}(S ; \pi)
$$

This completes the proof of the corollary.

\section{Proof of Corollary 3:}


We first show that $r_{\alpha}$ satisfies Path Independence. Pick any game $(N, v) \in \mathbb{V}$. Choose any $i, j \in N, S \subset N-i j$, and $T=N-(S+i j)$. Let $\pi \in \Pi_{T}$ be such that $\pi \equiv\left\{T_{1}, \ldots, T_{K}\right\}$. We want to show that

$$
v^{-i j}(S ; \pi)=v^{-j i}(S ; \pi)
$$

Indeed,

$$
\begin{aligned}
v^{-i j}(S ; \pi) & =\sum_{\pi^{\prime} \in \pi^{+j}(\pi)} \alpha\left(S ; \pi^{\prime}\right) v^{-i}\left(S ; \pi^{\prime}\right) \\
& =\sum_{\pi^{\prime} \in \pi^{+j}(\pi)} \alpha\left(S ; \pi^{\prime}\right)\left[\sum_{\pi^{\prime \prime} \in \pi^{+i}\left(\pi^{\prime}\right)} \alpha\left(S ; \pi^{\prime \prime}\right) v\left(S ; \pi^{\prime \prime}\right)\right]
\end{aligned}
$$

for some symmetric weight vector $\alpha$. Similarly,

$$
v^{-j i}(S ; \pi)=\sum_{\pi^{\prime} \in \pi^{+i}(\pi)} \alpha\left(S ; \pi^{\prime}\right)\left[\sum_{\pi^{\prime \prime} \in \pi^{+j}\left(\pi^{\prime}\right)} \alpha\left(S ; \pi^{\prime \prime}\right) v\left(S ; \pi^{\prime \prime}\right)\right]
$$

Since $\alpha$ is symmetric, for each $T_{k} \in \pi, \alpha\left(S ;\left(\pi \backslash\left\{T_{k}\right\}\right) \cup\left\{T_{k}+i\right\}\right)=\alpha\left(S ;\left(\pi \backslash\left\{T_{k}\right\}\right) \cup\right.$ $\left.\left\{T_{k}+j\right\}\right)$. Also, letting $\left.\pi^{1} \equiv\left(\pi \backslash\left\{T_{k}, T_{l}\right\}\right) \cup\left\{T_{k}+i, T_{l}+j\right\}\right)$, and $\pi^{2} \equiv(\pi-$ $\left.\left.\left\{T_{k}, T_{l}\right\}\right) \cup\left\{T_{k}+j, T_{l}+i\right\}\right)$, we have $\alpha\left(S ; \pi^{1}\right)=\alpha\left(S ; \pi^{2}\right)$. This is sufficient to show that $r_{\alpha}$ satisfies Path Independence.

Since the weight $\alpha(S ; \pi)$ depends only on the distribution of the cardinalities of the elements of $\pi$, it is straightforward to check that $r_{\alpha}$ satisfies Limited Independence. Translation Invariance follows similarly.

\section{Proof of Corollary 4:}

Let $\mathbb{P}$ be a partition of the universal set $\mathbb{N}$. By Theorem 5 and its proof it suffices to show that $r_{\mathbb{P}}$ satisfies both Path Independence and Regularity. Again, we omit any explicit reference to the operator in order to simplify the notation.

Fix any game $(N, v)$. To check that the $\mathbb{P}$-coordinate operator satisfies Path Independence, choose any $i, j \in N$, and $S \subset N-i j$. Let $\pi$ be any partition of $(S+i j)^{c}$. Now if $(S+i j)^{c} \cap \mathbb{P} \neq \pi$, then $v^{-i j}(S ; \pi)=0=v^{-j i}(S ; \pi)$; and if $(S+i j)^{c} \cap \mathbb{P}=\pi$, then $v^{-i j}(S ; \pi)=v\left(S ; S^{c} \cap \mathbb{P}\right)=v^{-j i}(S ; \pi)$. This shows that the $\mathbb{P}$-coordinate operator satisfies Path Independence.

In order to check Regularity, let $\varphi$ be a value, $(N, v) \in \mathbb{V}$ and $S \subset N$. For all $R \subseteq S$, we have by definition of $r_{\mathbb{P}}$

$$
\begin{aligned}
\left(v_{S}^{\varphi}\right)^{-S \backslash R}(R) & =v_{S}^{\varphi}(R ;(S \backslash R) \cap \mathbb{P}) \\
& =v\left(R \cup S^{c} ;(S \backslash R) \cap \mathbb{P}\right)-\sum_{k \in S^{c}} \varphi_{k}\left(R \cup S^{c}, v^{-S \backslash R}\right) \\
& =v^{-S \backslash R}\left(R \cup S^{c}\right)-\sum_{k \in S^{c}} \varphi_{k}\left(R \cup S^{c}, v^{-S \backslash R}\right),
\end{aligned}
$$

which is the desired conclusion. 


\section{Proof of Corollary 5:}

Suppose $r$ satisfies Path Independence, the Weak Dummy Axiom, Scale Invariance and Non-negativity. In view of Theorem 5 and its proof, it is sufficient to show that $r$ satisfies Regularity.

Let $w_{S}$ be the TU game associated with $v_{S}^{S h^{r}}$. Thus, $w_{S}(R)=\left(v_{S}^{S} h^{r}\right)^{-(S \backslash R)}(R)$. By repeated use of Theorem 2,

$$
\begin{aligned}
\left(v_{S}^{S h^{r}}\right)^{-(S \backslash R)}(R) & =\sum_{\pi \in \Pi_{S \backslash R}} \alpha_{\pi} v_{S}^{S h^{r}}(R ; \pi) \\
& =\sum_{\pi \in \Pi_{S \backslash R}} \alpha_{\pi}\left[v\left(R \cup S^{c} ; \pi\right)-\sum_{k \in S^{c}} S h_{k}^{r}\left(v^{-(S \backslash R)}\right)\right] \\
& =\sum_{\pi \in \Pi_{S \backslash R}} \alpha_{\pi} v\left(R \cup S^{c} ; \pi\right)-\left[\sum_{k \in S^{c}} S h_{k}^{r}\left(v^{-(S \backslash R)}\right)\right]\left[\sum_{\pi \in \Pi_{S \backslash R}} \alpha_{\pi}\right] \\
& =v^{-(S \backslash R)}\left(R \cup S^{c}\right)-\sum_{k \in S^{c}} S h_{k}^{r}\left(v^{-(S \backslash R)}\right)
\end{aligned}
$$

One can check that $\alpha_{\pi}=\theta^{(|S \backslash R|-|\pi|)} \Pi_{k=0}^{|P|-1}(1-k \theta)$ and $\sum_{\pi \in \Pi_{S \backslash R}} \alpha_{\pi}=1$. of course, the actual form of $\alpha_{\pi}$ is not important. What is important is that the restriction operator is linear, and that $\alpha_{\pi}$ does not depend upon $T$ in $v^{-(S \backslash R)}(T)$. 